\title{
A Gentle Introduction to Haskell 98
}

\author{
Paul Hudak \\ Yale University \\ Department of Computer Science
}

\author{
John Peterson \\ Yale University \\ Department of Computer Science
}

\author{
Joseph H. Fasel \\ University of California \\ Los Alamos National Laboratory
}

October, 1999

\section{Copyright (C) 1999 Paul Hudak, John Peterson and Joseph Fasel}

Permission is hereby granted, free of charge, to any person obtaining a copy of "A Gentle Introduction to Haskell" (the Text), to deal in the Text without restriction, including without limitation the rights to use, copy, modify, merge, publish, distribute, sublicense, and/or sell copies of the Text, and to permit persons to whom the Text is furnished to do so, subject to the following condition: The above copyright notice and this permission notice shall be included in all copies or substantial portions of the Text.

\section{Introduction}

Our purpose in writing this tutorial is not to teach programming, nor even to teach functional programming. Rather, it is intended to serve as a supplement to the Haskell Report [4], which is otherwise a rather dense technical exposition. Our goal is to provide a gentle introduction to Haskell for someone who has experience with at least one other language, preferably a functional language (even if only an "almost-functional" language such as ML or Scheme). If the reader wishes to learn more about the functional programming style, we highly recommend Bird's text Introduction to Functional Programming [1] or Davie's An Introduction to Functional Programming Systems Using Haskell [2]. For a useful survey of functional programming languages and techniques, including some of the language design principles used in Haskell, see [3].

The Haskell language has evolved significantly since its birth in 1987. This tutorial deals with Haskell 98. Older versions of the language are now obsolete; Haskell users are encouraged to use Haskell 98. There are also many extensions to Haskell 98 that have been widely implemented. These are not yet a formal part of the Haskell language and are not covered in this tutorial.

Our general strategy for introducing language features is this: motivate the idea, define some terms, give some examples, and then point to the Report for details. We suggest, however, that the reader completely ignore the details until the Gentle Introduction has been completely read. On the other hand, Haskell's Standard Prelude (in Appendix A of the Report and the standard libraries (found in the Library Report [5]) contain lots of useful examples of Haskell code; we encourage a thorough reading once this tutorial is completed. This will not only give the reader a feel for what real Haskell code looks like, but will also familiarize her with Haskell's standard set of predefined functions and types. 
Finally, the Haskell web site, http://haskell.org, has a wealth of information about the Haskell language and its implementations.

[We have also taken the course of not laying out a plethora of lexical syntax rules at the outset. Rather, we introduce them incrementally as our examples demand, and enclose them in brackets, as with this paragraph. This is in stark contrast to the organization of the Report, although the Report remains the authoritative source for details (references such as "§2.1" refer to sections in the Report).]

Haskell is a typeful programming language: ${ }^{1}$ types are pervasive, and the newcomer is best off becoming well aware of the full power and complexity of Haskell's type system from the outset. For those whose only experience is with relatively "untypeful" languages such as Perl, Tcl, or Scheme, this may be a difficult adjustment; for those familiar with Java, C, Modula, or even ML, the adjustment should be easier but still not insignificant, since Haskell's type system is different and somewhat richer than most. In any case, "typeful programming" is part of the Haskell programming experience, and cannot be avoided.

\section{Values, Types, and Other Goodies}

Because Haskell is a purely functional language, all computations are done via the evaluation of expressions (syntactic terms) to yield values (abstract entities that we regard as answers). Every value has an associated type. (Intuitively, we can think of types as sets of values.) Examples of expressions include atomic values such as the integer 5 , the character ' $a$ ', and the function $\backslash \mathrm{x} \rightarrow \mathrm{x}+1$, as well as structured values such as the list $[1,2,3]$ and the pair $\left({ }^{\prime} \mathrm{b} \mathrm{b}^{\prime}, 4\right)$.

Just as expressions denote values, type expressions are syntactic terms that denote type values (or just types). Examples of type expressions include the atomic types Integer (infiniteprecision integers), Char (characters), Integer->Integer (functions mapping Integer to Integer), as well as the structured types [Integer] (homogeneous lists of integers) and (Char, Integer) (character, integer pairs).

All Haskell values are "first-class" - they may be passed as arguments to functions, returned as results, placed in data structures, etc. Haskell types, on the other hand, are not first-class. Types in a sense describe values, and the association of a value with its type is called a typing. Using the examples of values and types above, we write typings as follows:

$$
\begin{aligned}
5 & :: \text { Integer } \\
\text { 'a' } & : \text { : Char } \\
\text { inc } & :: \text { Integer } \rightarrow \text { Integer } \\
{[1,2,3] } & :: \text { [Integer] } \\
\left({ }^{\prime} b, 4\right) & :: \text { (Char, Integer })
\end{aligned}
$$

The ": :" can be read "has type."

Functions in Haskell are normally defined by a series of equations. For example, the function inc can be defined by the single equation:

inc $\mathrm{n} \quad=\mathrm{n}+1$

An equation is an example of a declaration. Another kind of declaration is a type signature declaration (\$4.4.1), with which we can declare an explicit typing for inc: inc $:$ : Integer $\rightarrow$ Integer

We will have much more to say about function definitions in Section 3.

\footnotetext{
${ }^{1}$ Coined by Luca Cardelli.
} 
For pedagogical purposes, when we wish to indicate that an expression $e_{1}$ evaluates, or "reduces," to another expression or value $e_{2}$, we will write:

$$
e_{1} \quad \Rightarrow \quad e_{2}
$$

For example, note that:

$$
\text { inc (inc 3) } \Rightarrow 5
$$

Haskell's static type system defines the formal relationship between types and values (§4.1.3). The static type system ensures that Haskell programs are type safe; that is, that the programmer has not mismatched types in some way. For example, we cannot generally add together two characters, so the expression ' $a$ ' + ' $b$ ' is ill-typed. The main advantage of statically typed languages is well-known: All type errors are detected at compile-time. Not all errors are caught by the type system; an expression such as 1/0 is typable but its evaluation will result in an error at execution time. Still, the type system finds many program errors at compile time, aids the user in reasoning about programs, and also permits a compiler to generate more efficient code (for example, no run-time type tags or tests are required).

The type system also ensures that user-supplied type signatures are correct. In fact, Haskell's type system is powerful enough to allow us to avoid writing any type signatures at all $;^{2}$ we say that the type system infers the correct types for us. Nevertheless, judicious placement of type signatures such as that we gave for inc is a good idea, since type signatures are a very effective form of documentation and help bring programming errors to light.

[The reader will note that we have capitalized identifiers that denote specific types, such as Integer and Char, but not identifiers that denote values, such as inc. This is not just a convention: it is enforced by Haskell's lexical syntax. In fact, the case of the other characters matters, too: foo, fOo, and $f 00$ are all distinct identifiers.]

\section{$2.1 \quad$ Polymorphic Types}

Haskell also incorporates polymorphic types - types that are universally quantified in some way over all types. Polymorphic type expressions essentially describe families of types. For example, $(\forall a)[a]$ is the family of types consisting of, for every type a, the type of lists of a. Lists of integers (e.g. $[1,2,3])$, lists of characters (['a' , 'b' , 'c']), even lists of lists of integers, etc., are all members of this family. (Note, however, that [2, ' $b$ '] is not a valid example, since there is no single type that contains both 2 and ' $\mathrm{b}$ '.)

[Identifiers such as a above are called type variables, and are uncapitalized to distinguish them from specific types such as Int. Furthermore, since Haskell has only universally quantified types, there is no need to explicitly write out the symbol for universal quantification, and thus we simply write [a] in the example above. In other words, all type variables are implicitly universally quantified.]

Lists are a commonly used data structure in functional languages, and are a good vehicle for explaining the principles of polymorphism. The list $[1,2,3]$ in Haskell is actually shorthand for the list $1:(2:(3:[]))$, where [] is the empty list and : is the infix operator that adds its first argument to the front of its second argument (a list). ${ }^{3}$ Since : is right associative, we can also write this list as $1: 2: 3:[]$.

As an example of a user-defined function that operates on lists, consider the problem of counting the number of elements in a list:

\footnotetext{
${ }^{2}$ With a few exceptions to be described later.

${ }^{3}$ : and [] are like Lisp's cons and nil, respectively.
} 


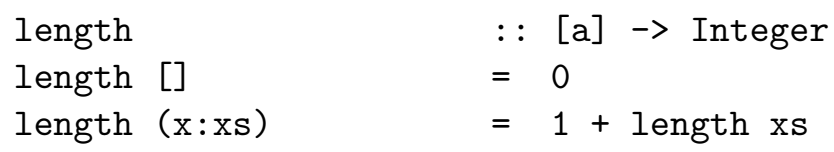

This definition is almost self-explanatory. We can read the equations as saying: "The length of the empty list is 0 , and the length of a list whose first element is $\mathrm{x}$ and remainder is $\mathrm{xs}$ is 1 plus the length of xs." (Note the naming convention used here; $x \mathrm{~s}$ is the plural of $\mathrm{x}$, and should be read that way.)

Although intuitive, this example highlights an important aspect of Haskell that is yet to be explained: pattern matching. The left-hand sides of the equations contain patterns such as [] and $\mathrm{x}$ :xs. In a function application these patterns are matched against actual parameters in a fairly intuitive way ([] only matches the empty list, and $\mathrm{x}$ : $\mathrm{xs}$ will successfully match any list with at least one element, binding $\mathrm{x}$ to the first element and $\mathrm{xs}$ to the rest of the list). If the match succeeds, the right-hand side is evaluated and returned as the result of the application. If it fails, the next equation is tried, and if all equations fail, an error results.

Defining functions by pattern matching is quite common in Haskell, and the user should become familiar with the various kinds of patterns that are allowed; we will return to this issue in Section 4.

The length function is also an example of a polymorphic function. It can be applied to a list containing elements of any type, for example [Integer], [Char], or [[Integer]].

$\begin{array}{lll}\text { length }[1,2,3] & \Rightarrow & 3 \\ \text { length ['a', 'b', 'c'] } & \Rightarrow & 3 \\ \text { length [[1], [2], [3] }] & \Rightarrow & 3\end{array}$

Here are two other useful polymorphic functions on lists that will be used later. Function head returns the first element of a list, function tail returns all but the first.

$\begin{array}{ll}\text { head } & ::[\mathrm{a}] \rightarrow \mathrm{a} \\ \text { head }(\mathrm{x}: \mathrm{xs}) & =\mathrm{x} \\ \text { tail } & ::[\mathrm{a}] \rightarrow[\mathrm{a}] \\ \text { tail }(\mathrm{x}: \mathrm{xs}) & =\mathrm{xs}\end{array}$

Unlike length, these functions are not defined for all possible values of their argument. A runtime error occurs when these functions are applied to an empty list.

With polymorphic types, we find that some types are in a sense strictly more general than others in the sense that the set of values they define is larger. For example, the type [a] is more general than [Char]. In other words, the latter type can be derived from the former by a suitable substitution for a. With regard to this generalization ordering, Haskell's type system possesses two important properties: First, every well-typed expression is guaranteed to have a unique principal type (explained below), and second, the principal type can be inferred automatically (§4.1.3). In comparison to a monomorphically typed language such as $\mathrm{C}$, the reader will find that polymorphism improves expressiveness, and type inference lessens the burden of types on the programmer.

An expression's or function's principal type is the least general type that, intuitively, "contains all instances of the expression". For example, the principal type of head is $[\mathrm{a}]->\mathrm{a} ;[\mathrm{b}]->\mathrm{a}$, a->a, or even a are correct types, but too general, whereas something like [Integer]->Integer is too specific. The existence of unique principal types is the hallmark feature of the HindleyMilner type system, which forms the basis of the type systems of Haskell, ML, Miranda, ${ }^{4}$ and several other (mostly functional) languages.

\footnotetext{
4 "Miranda" is a trademark of Research Software, Ltd.
} 


\subsection{User-Defined Types}

We can define our own types in Haskell using a data declaration, which we introduce via a series of examples (§4.2.1).

An important predefined type in Haskell is that of truth values:

data Bool

= False $\mid$ True

The type being defined here is Bool, and it has exactly two values: True and False. Type Bool is an example of a (nullary) type constructor, and True and False are (also nullary) data constructors (or just constructors, for short).

Similarly, we might wish to define a color type:

data Color $\quad=$ Red | Green | Blue | Indigo | Violet

Both Bool and Color are examples of enumerated types, since they consist of a finite number of nullary data constructors.

Here is an example of a type with just one data constructor:

data Point a

$=$ Pt $\mathrm{a}$ a

Because of the single constructor, a type like Point is often called a tuple type, since it is essentially just a cartesian product (in this case binary) of other types. ${ }^{5}$ In contrast, multiconstructor types, such as Bool and Color, are called (disjoint) union or sum types.

More importantly, however, Point is an example of a polymorphic type: for any type $t$, it defines the type of cartesian points that use $t$ as the coordinate type. The Point type can now be seen clearly as a unary type constructor, since from the type $t$ it constructs a new type Point $t$. (In the same sense, using the list example given earlier, [] is also a type constructor. Given any type $t$ we can "apply" [] to yield a new type [t]. The Haskell syntax allows [] $t$ to be written as [t]. Similarly, $\rightarrow$ is a type constructor: given two types $t$ and $u, t->u$ is the type of functions mapping elements of type $t$ to elements of type $u$.)

Note that the type of the binary data constructor Pt is a $\rightarrow$ a $\rightarrow$ Point $a$, and thus the following typings are valid:

$$
\begin{array}{ll}
\text { Pt } 2.03 .0 & :: \text { Point Float } \\
\text { Pt 'a' ' } b \text { ' } & \text { : Point Char } \\
\text { Pt True False } & : \text { : Point Bool }
\end{array}
$$

On the other hand, an expression such as Pt 'a' 1 is ill-typed because ' $a$ ' and 1 are of different types.

It is important to distinguish between applying a data constructor to yield a value, and applying a type constructor to yield a type; the former happens at run-time and is how we compute things in Haskell, whereas the latter happens at compile-time and is part of the type system's process of ensuring type safety.

[Type constructors such as Point and data constructors such as Pt are in separate namespaces. This allows the same name to be used for both a type constructor and data constructor, as in the following:

data Point a = Point a a

While this may seem a little confusing at first, it serves to make the link between a type and its data constructor more obvious.]

\footnotetext{
${ }^{5}$ Tuples are somewhat like records in other languages.
} 


\subsubsection{Recursive Types}

Types can also be recursive, as in the type of binary trees:

data Tree a = Leaf a $\mid$ Branch (Tree a) (Tree a)

Here we have defined a polymorphic binary tree type whose elements are either leaf nodes containing a value of type a, or internal nodes ("branches") containing (recursively) two subtrees.

When reading data declarations such as this, remember again that Tree is a type constructor, whereas Branch and Leaf are data constructors. Aside from establishing a connection between these constructors, the above declaration is essentially defining the following types for Branch and Leaf:

Branch $\quad::$ Tree a $\rightarrow$ Tree a $\rightarrow$ Tree a

Leaf $\quad::$ a $\rightarrow$ Tree a

With this example we have defined a type sufficiently rich to allow defining some interesting (recursive) functions that use it. For example, suppose we wish to define a function fringe that returns a list of all the elements in the leaves of a tree from left to right. It's usually helpful to write down the type of new functions first; in this case we see that the type should be Tree a $\rightarrow$ [a]. That is, fringe is a polymorphic function that, for any type a, maps trees of a into lists of a. A suitable definition follows:

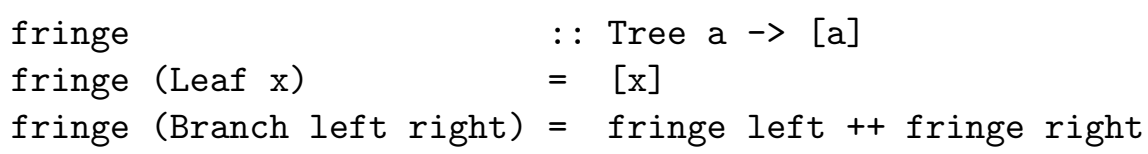

Here ++ is the infix operator that concatenates two lists (its full definition will be given in Section 9.1). As with the length example given earlier, the fringe function is defined using pattern matching, except that here we see patterns involving user-defined constructors: Leaf and Branch. [Note that the formal parameters are easily identified as the ones beginning with lower-case letters.]

\subsection{Type Synonyms}

For convenience, Haskell provides a way to define type synonyms; i.e. names for commonly used types. Type synonyms are created using a type declaration (§4.2.2). Here are several examples:

$$
\begin{array}{ll}
\text { type String } & =[\text { Char }] \\
\text { type Person } & =\text { (Name, Address }) \\
\text { type Name } & =\text { String } \\
\text { data Address } & =\text { None | Addr String }
\end{array}
$$

Type synonyms do not define new types, but simply give new names for existing types. For example, the type Person $\rightarrow$ Name is precisely equivalent to (String, Address) -> String. The new names are often shorter than the types they are synonymous with, but this is not the only purpose of type synonyms: they can also improve readability of programs by being more mnemonic; indeed, the above examples highlight this. We can even give new names to polymorphic types:

type AssocList a b = $[(a, b)]$

This is the type of "association lists" which associate values of type a with those of type b. 


\subsection{Built-in Types Are Not Special}

Earlier we introduced several "built-in" types such as lists, tuples, integers, and characters. We have also shown how new user-defined types can be defined. Aside from special syntax, are the built-in types in any way more special than the user-defined ones? The answer is no. The special syntax is for convenience and for consistency with historical convention, but has no semantic consequences.

We can emphasize this point by considering what the type declarations would look like for these built-in types if in fact we were allowed to use the special syntax in defining them. For example, the Char type might be written as:

data Char

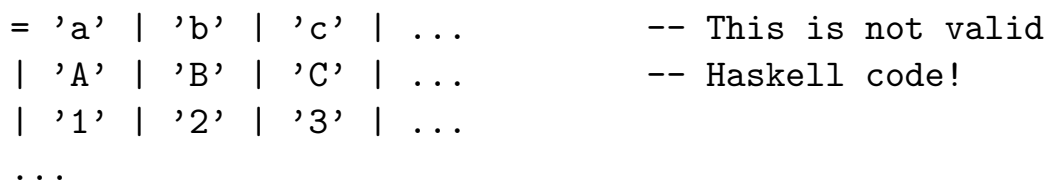

These constructor names are not syntactically valid; to fix them we would have to write something like:

data Char

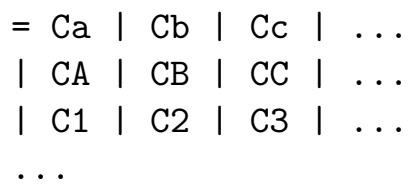

Even though these constructors are more concise, they are quite unconventional for representing characters.

In any case, writing "pseudo-Haskell" code in this way helps us to see through the special syntax. We see now that Char is just an enumerated type consisting of a large number of nullary constructors. Thinking of Char in this way makes it clear that we can pattern-match against characters in function definitions, just as we would expect to be able to do so for any of a type's constructors.

[This example also demonstrates the use of comments in Haskell; the characters -- and all subsequent characters to the end of the line are ignored. Haskell also permits nested comments which have the form $\{-\ldots-\}$ and can appear anywhere (§2.2).]

Similarly, we could define Int (fixed precision integers) and Integer by:

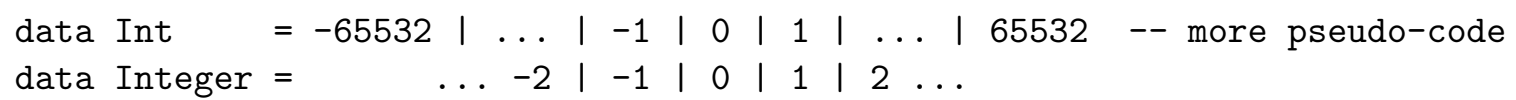

where -65532 and 65532 , say, are the maximum and minimum fixed precision integers for a given implementation. Int is a much larger enumeration than Char, but it's still finite! In contrast, the pseudo-code for Integer is intended to convey an infinite enumeration.

Tuples are also easy to define playing this game:

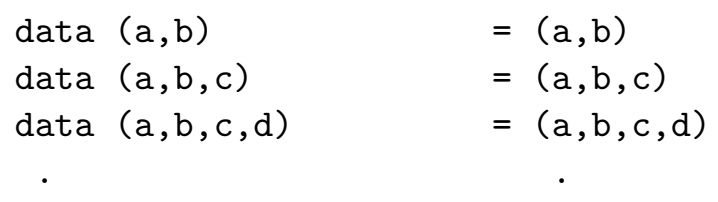

Each declaration above defines a tuple type of a particular length, with (...) playing a role in both the expression syntax (as data constructor) and type-expression syntax (as type constructor). The vertical dots after the last declaration are intended to convey an infinite number of such declarations, reflecting the fact that tuples of all lengths are allowed in Haskell. 
Lists are also easily handled, and more interestingly, they are recursive:
data $[a]$
$=[] \quad \mathrm{a}:[\mathrm{a}]$
-- more pseudo-code

We can now see clearly what we described about lists earlier: [] is the empty list, and : is the infix list constructor; thus $[1,2,3]$ must be equivalent to the list $1: 2: 3:[]$. (: is right associative.) The type of [] is $[a]$, and the type of $:$ is $a->[a]->[a]$.

[The way ":" is defined here is actually legal syntax-infix constructors are permitted in data declarations, and are distinguished from infix operators (for pattern-matching purposes) by the fact that they must begin with a ":" (a property trivially satisfied by ":").]

At this point the reader should note carefully the differences between tuples and lists, which the above definitions make abundantly clear. In particular, note the recursive nature of the list type whose elements are homogeneous and of arbitrary length, and the non-recursive nature of a (particular) tuple type whose elements are heterogeneous and of fixed length. The typing rules for tuples and lists should now also be clear:

For $\left(e_{1}, e_{2}, \ldots, e_{n}\right), n \geq 2$, if $t_{i}$ is the type of $e_{i}$, then the type of the tuple is $\left(t_{1}, t_{2}, \ldots, t_{n}\right)$. $[t]$.

For $\left[e_{1}, e_{2}, \ldots, e_{n}\right], n \geq 0$, each $e_{i}$ must have the same type $t$, and the type of the list is

\subsubsection{List Comprehensions and Arithmetic Sequences}

As with Lisp dialects, lists are pervasive in Haskell, and as with other functional languages, there is yet more syntactic sugar to aid in their creation. Aside from the constructors for lists just discussed, Haskell provides an expression known as a list comprehension that is best explained by example:

$[f x \mid x<-x s]$

This expression can intuitively be read as "the list of all $f \mathrm{x}$ such that $\mathrm{x}$ is drawn from xs." The similarity to set notation is not a coincidence. The phrase $\mathrm{x}<-\mathrm{xs}$ is called a generator, of which more than one is allowed, as in:

$$
[(x, y) \mid x<-x s, y<-y s]
$$

This list comprehension forms the cartesian product of the two lists xs and ys. The elements are selected as if the generators were "nested" from left to right (with the rightmost generator varying fastest); thus, if $x s$ is $[1,2]$ and ys is $[3,4]$, the result is $[(1,3),(1,4),(2,3),(2,4)]$.

Besides generators, boolean expressions called guards are permitted. Guards place constraints on the elements generated. For example, here is a concise definition of everybody's favorite sorting algorithm:

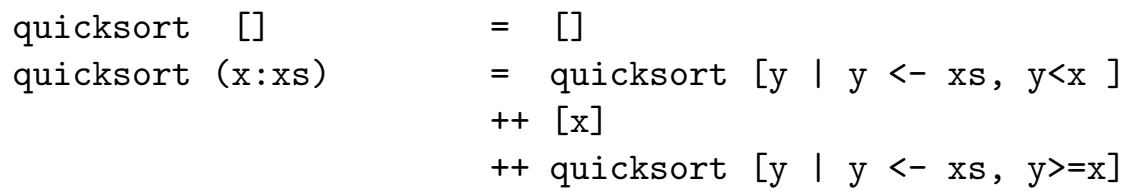

To further support the use of lists, Haskell has special syntax for arithmetic sequences, which are best explained by a series of examples:

$$
\begin{array}{llll}
{[1 \ldots 10]} & \Rightarrow & {[1,2,3,4,5,6,7,8,9,10]} \\
{[1,3 \ldots 10]} & \Rightarrow & {[1,3,5,7,9]} \\
{[1,3 \ldots]} & \Rightarrow & {[1,3,5,7,9, \ldots \quad \text { (infinite sequence) }}
\end{array}
$$

More will be said about arithmetic sequences in Section 8.2, and "infinite lists" in Section 3.4. 


\subsubsection{Strings}

As another example of syntactic sugar for built-in types, we note that the literal string "hello" is actually shorthand for the list of characters [' $h$ ', 'e', 'l', 'l', 'o']. Indeed, the type of "hello" is String, where String is a predefined type synonym (that we gave as an earlier example):

type String $\quad=[$ Char $]$

This means we can use predefined polymorphic list functions to operate on strings. For example:

"hello" ++ " world" $\Rightarrow$ "hello world"

\section{Functions}

Since Haskell is a functional language, one would expect functions to play a major role, and indeed they do. In this section, we look at several aspects of functions in Haskell.

First, consider this definition of a function which adds its two arguments:

add $\quad:$ : Integer $\rightarrow$ Integer $\rightarrow$ Integer

add $\mathrm{x}$ y $\quad=\mathrm{x}+\mathrm{y}$

This is an example of a curried function. ${ }^{6}$ An application of add has the form add $e_{1} e_{2}$, and is equivalent to (add $e_{1}$ ) $e_{2}$, since function application associates to the left. In other words, applying add to one argument yields a new function which is then applied to the second argument. This is consistent with the type of add, Integer->Integer->Integer, which is equivalent to Integer-> (Integer->Integer); i.e. -> associates to the right. Indeed, using add, we can define inc in a different way from earlier:

inc $\quad=$ add 1

This is an example of the partial application of a curried function, and is one way that a function can be returned as a value. Let's consider a case in which it's useful to pass a function as an argument. The well-known map function is a perfect example:

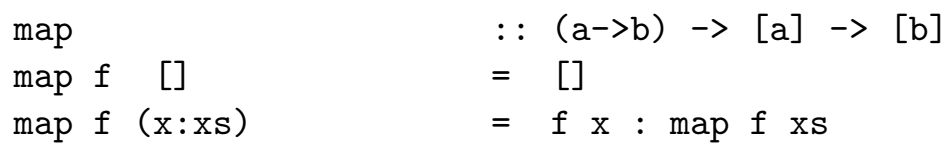

[Function application has higher precedence than any infix operator, and thus the right-hand side of the second equation parses as $(f \mathrm{x})$ : (map $\mathrm{f} x \mathrm{~s})$.] The map function is polymorphic and its type indicates clearly that its first argument is a function; note also that the two a's must be instantiated with the same type (likewise for the b's). As an example of the use of map, we can increment the elements in a list:

$$
\operatorname{map}(\operatorname{add} 1)[1,2,3] \quad \Rightarrow \quad[2,3,4]
$$

These examples demonstrate the first-class nature of functions, which when used in this way are usually called higher-order functions.

\footnotetext{
${ }^{6}$ The name curry derives from the person who popularized the idea: Haskell Curry. To get the effect of an uncurried function, we could use a tuple, as in:

add $(\mathrm{x}, \mathrm{y})$

$=\mathrm{x}+\mathrm{y}$

But then we see that this version of add is really just a function of one argument!
} 


\subsection{Lambda Abstractions}

Instead of using equations to define functions, we can also define them "anonymously" via a lambda abstraction. For example, a function equivalent to inc could be written as $\backslash \mathrm{x} \rightarrow \mathrm{x}+1$. Similarly, the function add is equivalent to $\backslash \mathrm{x} \rightarrow \backslash \mathrm{y} \rightarrow \mathrm{x}+\mathrm{y}$. Nested lambda abstractions such as this may be written using the equivalent shorthand notation $\backslash \mathrm{x} y \rightarrow \mathrm{x}+\mathrm{y}$. In fact, the equations:

$\begin{array}{ll}\text { inc } \mathrm{x} & =\mathrm{x}+1 \\ \text { add } \mathrm{x} y & =\mathrm{x}+\mathrm{y}\end{array}$

are really shorthand for:

$$
\begin{array}{lll}
\text { inc } & =\backslash x & \rightarrow x+1 \\
\text { add } & & =\backslash x \quad y \quad>x+y
\end{array}
$$

We will have more to say about such equivalences later.

In general, given that $\mathrm{x}$ has type $t_{1}$ and exp has type $t_{2}$, then $\backslash \mathrm{x}->\exp$ has type $t_{1}->t_{2}$.

\subsection{Infix Operators}

Infix operators are really just functions, and can also be defined using equations. For example, here is a definition of a list concatenation operator:

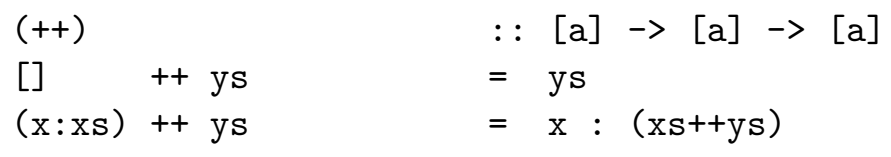

[Lexically, infix operators consist entirely of "symbols," as opposed to normal identifiers which are alphanumeric (§2.4). Haskell has no prefix operators, with the exception of minus (-), which is both infix and prefix.]

As another example, an important infix operator on functions is that for function composition:
(.)
$::(b->c)->(a->b) \rightarrow(a->c)$
f. $g$
$=\backslash x \rightarrow f(g x)$

\subsubsection{Sections}

Since infix operators are really just functions, it makes sense to be able to partially apply them as well. In Haskell the partial application of an infix operator is called a section. For example:

$$
\begin{array}{ccc}
(\mathrm{x}+) & \equiv & \backslash \mathrm{y} \rightarrow \mathrm{x}+\mathrm{y} \\
(+\mathrm{y}) & \equiv & \backslash \mathrm{x} \rightarrow \mathrm{x}+\mathrm{y} \\
(+) & \equiv & \backslash \mathrm{x} \mathrm{y} \rightarrow \mathrm{x}+\mathrm{y}
\end{array}
$$

[The parentheses are mandatory.]

The last form of section given above essentially coerces an infix operator into an equivalent functional value, and is handy when passing an infix operator as an argument to a function, as in map (+) $[1,2,3]$ (the reader should verify that this returns a list of functions!). It is also necessary when giving a function type signature, as in the examples of (++) and (.) given earlier.

We can now see that add defined earlier is just $(+)$, and inc is just $(+1)$ ! Indeed, these definitions would do just fine: 
inc

add

$=(+1)$

$=(+)$

We can coerce an infix operator into a functional value, but can we go the other way? Yes - we simply enclose an identifier bound to a functional value in backquotes. For example, $\mathrm{x}$ 'add' $\mathrm{y}$ is the same as add $\mathrm{x} y .{ }^{7}$ Some functions read better this way. An example is the predefined list membership predicate elem; the expression $\mathrm{x}$ ' $\mathrm{elem}$ ' $\mathrm{xs}$ can be read intuitively as " $\mathrm{x}$ is an element of $\mathrm{xs} . "$

[There are some special rules regarding sections involving the prefix/infix operator -; see $(\S 3.5, \S 3.4)$.

At this point, the reader may be confused at having so many ways to define a function! The decision to provide these mechanisms partly reflects historical conventions, and partly reflects the desire for consistency (for example, in the treatment of infix vs. regular functions).

\subsubsection{Fixity Declarations}

A fixity declaration can be given for any infix operator or constructor (including those made from ordinary identifiers, such as 'elem'). This declaration specifies a precedence level from 0 to 9 (with 9 being the strongest; normal application is assumed to have a precedence level of 10), and left-, right-, or non-associativity. For example, the fixity declarations for ++ and . are:

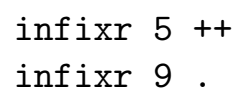

Both of these specify right-associativity, the first with a precedence level of 5 , the other 9 . Left associativity is specified via infixl, and non-associativity by infix. Also, the fixity of more than one operator may be specified with the same fixity declaration. If no fixity declaration is given for a particular operator, it defaults to infixl 9. (See $\S 5.9$ for a detailed definition of the associativity rules.)

\subsection{Functions are Non-strict}

Suppose bot is defined by:

bot $\quad$ bot

In other words, bot is a non-terminating expression. Abstractly, we denote the value of a nonterminating expression as $\perp$ (read "bottom"). Expressions that result in some kind of a run-time error, such as 1/0, also have this value. Such an error is not recoverable: programs will not continue past these errors. Errors encountered by the I/O system, such as an end-of-file error, are recoverable and are handled in a different manner. (Such an I/O error is really not an error at all but rather an exception. Much more will be said about exceptions in Section 7.)

A function $\mathrm{f}$ is said to be strict if, when applied to a nonterminating expression, it also fails to terminate. In other words, $f$ is strict iff the value of $f$ bot is $\perp$. For most programming languages, all functions are strict. But this is not so in Haskell. As a simple example, consider const1, the constant 1 function, defined by:

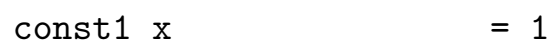

The value of const1 bot in Haskell is 1 . Operationally speaking, since const1 does not "need"

\footnotetext{
${ }^{7}$ Note carefully that add is enclosed in backquotes, not apostrophes as used in the syntax of characters; i.e. 'f ' is a character, whereas ' $f$ ' is an infix operator. Fortunately, most ASCII terminals distinguish these much better than the font used in this manuscript.
} 
the value of its argument, it never attempts to evaluate it, and thus never gets caught in a nonterminating computation. For this reason, non-strict functions are also called "lazy functions", and are said to evaluate their arguments "lazily", or "by need".

Since error and nonterminating values are semantically the same in Haskell, the above argument also holds for errors. For example, const1 (1/0) also evaluates properly to 1.

Non-strict functions are extremely useful in a variety of contexts. The main advantage is that they free the programmer from many concerns about evaluation order. Computationally expensive values may be passed as arguments to functions without fear of them being computed if they are not needed. An important example of this is a possibly infinite data structure.

Another way of explaining non-strict functions is that Haskell computes using definitions rather than the assignments found in traditional languages. Read a declaration such as

$\mathrm{V}$

$=1 / 0$

as 'define $\mathrm{v}$ as $1 / 0$ ' instead of 'compute $1 / 0$ and store the result in v'. Only if the value (definition) of $\mathrm{v}$ is needed will the division by zero error occur. By itself, this declaration does not imply any computation. Programming using assignments requires careful attention to the ordering of the assignments: the meaning of the program depends on the order in which the assignments are executed. Definitions, in contrast, are much simpler: they can be presented in any order without affecting the meaning of the program.

\section{4 "Infinite" Data Structures}

One advantage of the non-strict nature of Haskell is that data constructors are non-strict, too. This should not be surprising, since constructors are really just a special kind of function (the distinguishing feature being that they can be used in pattern matching). For example, the constructor for lists, $(:)$, is non-strict.

Non-strict constructors permit the definition of (conceptually) infinite data structures. Here is an infinite list of ones:

ones $\quad=1$ : ones

Perhaps more interesting is the function numsFrom:

numsFrom $\mathrm{n} \quad \mathrm{n}$ : numsFrom $(\mathrm{n}+1)$

Thus numsFrom $\mathrm{n}$ is the infinite list of successive integers beginning with $\mathrm{n}$. From it we can construct an infinite list of squares:

squares $\quad=\operatorname{map}(\neg 2)$ (numsfrom 0 )

(Note the use of a section; “ is the infix exponentiation operator.)

Of course, eventually we expect to extract some finite portion of the list for actual computation, and there are lots of predefined functions in Haskell that do this sort of thing: take, takeWhile, filter, and others. The definition of Haskell includes a large set of built-in functions and types - this is called the "Standard Prelude". The complete Standard Prelude is included in Appendix A of the Haskell report; see the portion named PreludeList for many useful functions involving lists. For example, take removes the first $\mathrm{n}$ elements from a list:

$$
\text { take } 5 \text { squares } \Rightarrow[0,1,4,9,16]
$$

The definition of ones above is an example of a circular list. In most circumstances laziness has an important impact on efficiency, since an implementation can be expected to implement the list as a true circular structure, thus saving space. 


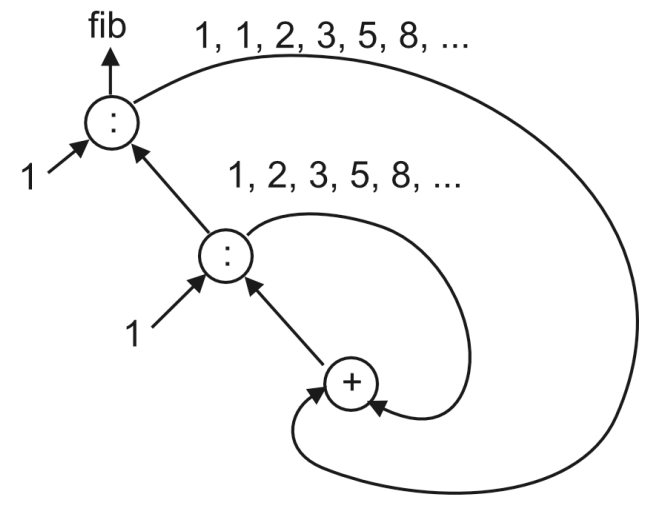

Figure 1: Circular Fibonacci Sequence

For another example of the use of circularity, the Fibonacci sequence can be computed efficiently as the following infinite sequence:

fib

$$
=1: 1:[a+b \mid(a, b)<- \text { zip fib (tail fib) ] }
$$

where zip is a Standard Prelude function that returns the pairwise interleaving of its two list arguments:

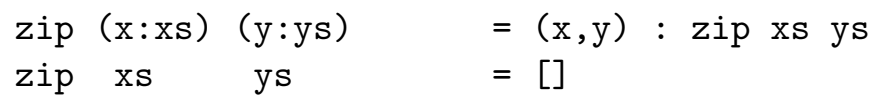

Note how $f i b$, an infinite list, is defined in terms of itself, as if it were "chasing its tail." Indeed, we can draw a picture of this computation as shown in Figure 1.

For another application of infinite lists, see Section 4.4.

\subsection{The Error Function}

Haskell has a built-in function called error whose type is String->a. This is a somewhat odd function: From its type it looks as if it is returning a value of a polymorphic type about which it knows nothing, since it never receives a value of that type as an argument!

In fact, there is one value "shared" by all types: $\perp$. Indeed, semantically that is exactly what value is always returned by error (recall that all errors have value $\perp$ ). However, we can expect that a reasonable implementation will print the string argument to error for diagnostic purposes. Thus this function is useful when we wish to terminate a program when something has "gone wrong." For example, the actual definition of head taken from the Standard Prelude is:

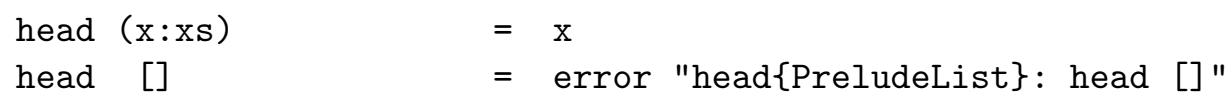

\section{Case Expressions and Pattern Matching}

Earlier we gave several examples of pattern matching in defining functions - for example length and fringe. In this section we will look at the pattern-matching process in greater detail $(\S 3.17) .{ }^{8}$

\footnotetext{
${ }^{8}$ Pattern matching in Haskell is different from that found in logic programming languages such as Prolog; in particular, it can be viewed as "one-way" matching, whereas Prolog allows "two-way" matching (via unification), along with implicit backtracking in its evaluation mechanism.
} 
Patterns are not "first-class;" there is only a fixed set of different kinds of patterns. We have already seen several examples of data constructor patterns; both length and fringe defined earlier use such patterns, the former on the constructors of a "built-in" type (lists), the latter on a user-defined type (Tree). Indeed, matching is permitted using the constructors of any type, user-defined or not. This includes tuples, strings, numbers, characters, etc. For example, here's a contrived function that matches against a tuple of "constants:"

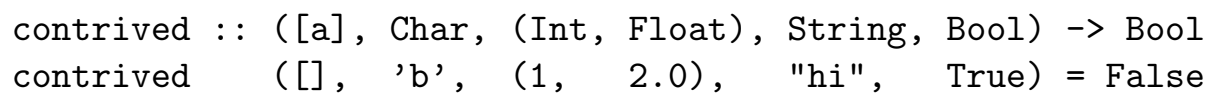

This example also demonstrates that nesting of patterns is permitted (to arbitrary depth).

Technically speaking, formal parameters ${ }^{9}$ are also patterns - it's just that they never fail to match a value. As a "side effect" of the successful match, the formal parameter is bound to the value it is being matched against. For this reason patterns in any one equation are not allowed to have more than one occurrence of the same formal parameter (a property called linearity $\S 3.17, \S 3.3, \S 4.4 .2)$.

Patterns such as formal parameters that never fail to match are said to be irrefutable, in contrast to refutable patterns which may fail to match. The pattern used in the contrived example above is refutable. There are three other kinds of irrefutable patterns, two of which we will introduce now (the other we will delay until Section 4.4).

As-patterns. Sometimes it is convenient to name a pattern for use on the right-hand side of an equation. For example, a function that duplicates the first element in a list might be written as:

$$
f(x: x s) \quad=x: x: x s
$$

(Recall that ":" associates to the right.) Note that x:xs appears both as a pattern on the lefthand side, and an expression on the right-hand side. To improve readability, we might prefer to write $\mathrm{x}: \mathrm{xs}$ just once, which we can achieve using an as-pattern as follows: ${ }^{10}$

$$
f \mathrm{~s} @(\mathrm{x}: \mathrm{xs}) \quad \mathrm{x}: \mathrm{s}
$$

Technically speaking, as-patterns always result in a successful match, although the sub-pattern (in this case $\mathbf{x}: \mathbf{x s}$ ) could, of course, fail.

Wild-cards. Another common situation is matching against a value we really care nothing about. For example, the functions head and tail defined in Section 2.1 can be rewritten as:

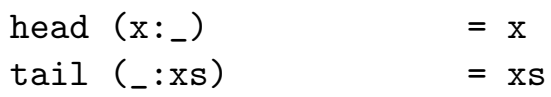

in which we have "advertised" the fact that we don't care what a certain part of the input is. Each wild-card independently matches anything, but in contrast to a formal parameter, each binds nothing; for this reason more than one is allowed in an equation.

\subsection{Pattern-Matching Semantics}

So far we have discussed how individual patterns are matched, how some are refutable, some are irrefutable, etc. But what drives the overall process? In what order are the matches attempted? What if none succeeds? This section addresses these questions.

\footnotetext{
${ }^{9}$ The Report calls these variables.

${ }^{10}$ Another advantage to doing this is that a naive implementation might completely reconstruct $\mathrm{x}: \mathrm{xs}$ rather than re-use the value being matched against.
} 
Pattern matching can either fail, succeed or diverge. A successful match binds the formal parameters in the pattern. Divergence occurs when a value needed by the pattern contains an error $(\perp)$. The matching process itself occurs "top-down, left-to-right." Failure of a pattern anywhere in one equation results in failure of the whole equation, and the next equation is then tried. If all equations fail, the value of the function application is $\perp$, and results in a run-time error.

For example, if $[1,2]$ is matched against $[0$, bot $]$, then 1 fails to match 0 , so the result is a failed match. (Recall that bot, defined earlier, is a variable bound to $\perp$.) But if $[1,2]$ is matched against [bot, 0], then matching 1 against bot causes divergence (i.e. $\perp$ ).

The other twist to this set of rules is that top-level patterns may also have a boolean guard, as in this definition of a function that forms an abstract version of a number's sign:

\begin{tabular}{r|ll}
$\operatorname{sign} x \mid$ & $x>0$ & $=1$ \\
$\mid x==0$ & $=$ & 0 \\
$\mid x<0$ & $=$ & -1
\end{tabular}

Note that a sequence of guards may be provided for the same pattern; as with patterns, they are evaluated top-down, and the first that evaluates to True results in a successful match.

\subsection{An Example}

The pattern-matching rules can have subtle effects on the meaning of functions. For example, consider this definition of take:

$$
\begin{aligned}
& \text { take } 0 \text { - }=[] \\
& \text { take - [ ] } \quad=[] \\
& \text { take } \mathrm{n}(\mathrm{x}: \mathrm{xs})=\mathrm{x} \text { : take }(\mathrm{n}-1) \mathrm{xs}
\end{aligned}
$$

and this slightly different version (the first 2 equations have been reversed):

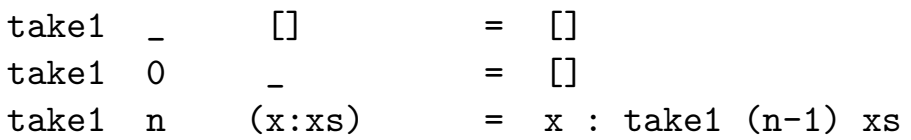

Now note the following:

$\begin{array}{llll}\text { take } 0 \text { bot } & \Rightarrow & {[]} \\ \text { take1 } 0 \text { bot } & \Rightarrow & \perp \\ \text { take bot [] } & \Rightarrow & \perp \\ \text { take1 bot [] } & \Rightarrow & {[]}\end{array}$

We see that take is "more defined" with respect to its second argument, whereas take1 is more defined with respect to its first. It is difficult to say in this case which definition is better. Just remember that in certain applications, it may make a difference. (The Standard Prelude includes a definition corresponding to take.)

\subsection{Case Expressions}

Pattern matching provides a way to "dispatch control" based on structural properties of a value. In many circumstances we don't wish to define a function every time we need to do this, but so far we have only shown how to do pattern matching in function definitions. Haskell's case expression provides a way to solve this problem. Indeed, the meaning of pattern matching in function definitions is specified in the Report in terms of case expressions, which are considered 
more primitive. In particular, a function definition of the form:

$$
\begin{aligned}
& \text { f } p_{11} \ldots p_{1 k}=e_{1} \\
& \ldots \\
& \text { f } p_{n 1} \ldots \\
& p_{n k}=e_{n}
\end{aligned}
$$

where each $p_{i j}$ is a pattern, is semantically equivalent to:

$$
\begin{aligned}
\mathrm{f} \mathrm{x} 1 \mathrm{x} 2 \ldots \mathrm{xk}=\operatorname{case}(\mathrm{x} 1, \ldots, \mathrm{xk}) \text { of } & \left(p_{11}, \ldots, p_{1 k}\right) \rightarrow e_{1} \\
& \ldots \\
& \left(p_{n 1}, \ldots, p_{n k}\right) \rightarrow e_{n}
\end{aligned}
$$

where the $\mathrm{xi}$ are new identifiers. (For a more general translation that includes guards, see §4.4.2.) For example, the definition of take given earlier is equivalent to:

$$
\begin{aligned}
& \text { take } \mathrm{m} \text { ys } \quad=\text { case }(\mathrm{m}, \mathrm{ys}) \text { of } \\
& \left(0,{ }_{-}\right) \quad \rightarrow \quad[] \\
& \left({ }_{-},[]\right) \quad \rightarrow \quad[] \\
& (\mathrm{n}, \mathrm{x}: \mathrm{xs}) \quad \rightarrow \mathrm{x} \text { : take }(\mathrm{n}-1) \mathrm{xs}
\end{aligned}
$$

A point not made earlier is that, for type correctness, the types of the right-hand sides of a case expression or set of equations comprising a function definition must all be the same; more precisely, they must all share a common principal type.

The pattern-matching rules for case expressions are the same as we have given for function definitions, so there is really nothing new to learn here, other than to note the convenience that case expressions offer. Indeed, there's one use of a case expression that is so common that it has special syntax: the conditional expression. In Haskell, conditional expressions have the familiar form:

$$
\text { if } e_{1} \text { then } e_{2} \text { else } e_{3}
$$

which is really short-hand for:

$$
\begin{array}{lll}
\text { case } e_{1} \text { of } & \text { True } & \rightarrow e_{2} \\
& \text { False } & \rightarrow e_{3}
\end{array}
$$

From this expansion it should be clear that $e_{1}$ must have type Bool, and $e_{2}$ and $e_{3}$ must have the same (but otherwise arbitrary) type. In other words, if-then-else when viewed as a function has type Bool->a->a->a.

\subsection{Lazy Patterns}

There is one other kind of pattern allowed in Haskell. It is called a lazy pattern, and has the form pat. Lazy patterns are irrefutable: matching a value $v$ against ${ }^{p a t}$ always succeeds, regardless of pat. Operationally speaking, if an identifier in pat is later "used" on the right-hand-side, it will be bound to that portion of the value that would result if $v$ were to successfully match pat, and $\perp$ otherwise.

Lazy patterns are useful in contexts where infinite data structures are being defined recursively. For example, infinite lists are an excellent vehicle for writing simulation programs, and in this context the infinite lists are often called streams. Consider the simple case of simulating the interactions between a server process server and a client process client, where client sends a sequence of requests to server, and server replies to each request with some kind of response. This situation is shown pictorially in Figure 2. (Note that client also takes an initial message 


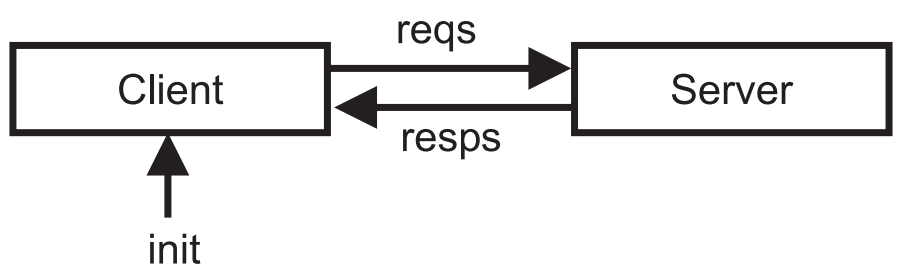

Figure 2: Client-Server Simulation

as argument.) Using streams to simulate the message sequences, the Haskell code corresponding to this diagram is:

$$
\begin{array}{ll}
\text { reqs } & =\text { client init resps } \\
\text { resps } & =\text { server reqs }
\end{array}
$$

These recursive equations are a direct lexical transliteration of the diagram.

Let us further assume that the structure of the server and client look something like this:

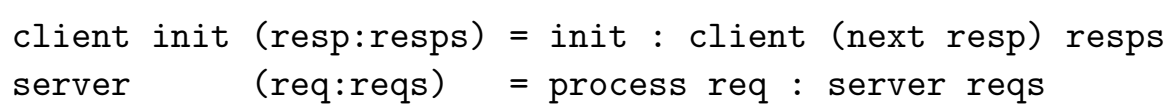

where we assume that next is a function that, given a response from the server, determines the next request, and process is a function that processes a request from the client, returning an appropriate response.

Unfortunately, this program has a serious problem: it will not produce any output! The problem is that client, as used in the recursive setting of reqs and resps, attempts a match on the response list before it has submitted its first request! In other words, the pattern matching is being done "too early." One way to fix this is to redefine client as follows:

client init resps = init : client (next (head resps)) (tail resps)

Although workable, this solution does not read as well as that given earlier. A better solution is to use a lazy pattern:

client init (resp:resps) = init : client (next resp) resps

Because lazy patterns are irrefutable, the match will immediately succeed, allowing the initial request to be "submitted", in turn allowing the first response to be generated; the engine is now "primed", and the recursion takes care of the rest.

As an example of this program in action, if we define:

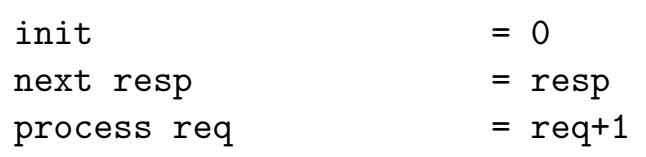

then we see that:

$$
\text { take } 10 \text { reqs } \Rightarrow[0,1,2,3,4,5,6,7,8,9]
$$

As another example of the use of lazy patterns, consider the definition of Fibonacci given earlier:

fib $\quad=1: 1:[a+b \mid(a, b)<-$ zip fib (tail fib) ]

We might try rewriting this using an as-pattern:

$$
\text { fib@(1:tfib) = } 1: 1:[a+b \mid(a, b)<- \text { zip fib tfib ] }
$$

This version of $f i b$ has the (small) advantage of not using tail on the right-hand side, since it is available in "destructured" form on the left-hand side as tfib. 
[This kind of equation is called a pattern binding because it is a top-level equation in which the entire left-hand side is a pattern; i.e. both $f i b$ and $t f i b$ become bound within the scope of the declaration.]

Now, using the same reasoning as earlier, we should be led to believe that this program will not generate any output. Curiously, however, it does, and the reason is simple: in Haskell, pattern bindings are assumed to have an implicit in front of them, reflecting the most common behavior expected of pattern bindings, and avoiding some anomalous situations which are beyond the scope of this tutorial. Thus we see that lazy patterns play an important role in Haskell, if only implicitly.

\subsection{Lexical Scoping and Nested Forms}

It is often desirable to create a nested scope within an expression, for the purpose of creating local bindings not seen elsewhere - i.e. some kind of "block-structuring" form. In Haskell there are two ways to achieve this:

Let Expressions. Haskell's let expressions are useful whenever a nested set of bindings is required. As a simple example, consider:

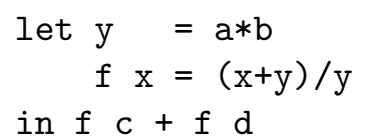

The set of bindings created by a let expression is mutually recursive, and pattern bindings are treated as lazy patterns (i.e. they carry an implicit ). The only kind of declarations permitted are type signatures, function bindings, and pattern bindings.

Where Clauses. Sometimes it is convenient to scope bindings over several guarded equations, which requires a where clause:

$$
\begin{array}{clll}
f \times \mathrm{x} \quad \mathrm{y}>\mathrm{z} & = & \ldots \\
\mathrm{y}==\mathrm{z} & = & \ldots \\
\mathrm{y}<\mathrm{z} & = & \ldots
\end{array}
$$

Note that this cannot be done with a let expression, which only scopes over the expression which it encloses. A where clause is only allowed at the top level of a set of equations or case expression. The same properties and constraints on bindings in let expressions apply to those in where clauses.

These two forms of nested scope seem very similar, but remember that a let expression is an expression, whereas a where clause is not-it is part of the syntax of function declarations and case expressions.

\subsection{Layout}

The reader may have been wondering how it is that Haskell programs avoid the use of semicolons, or some other kind of terminator, to mark the end of equations, declarations, etc. For example, consider this let expression from the last section:

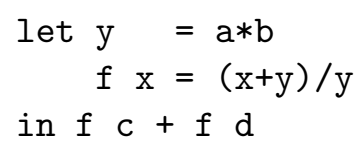


How does the parser know not to parse this as:

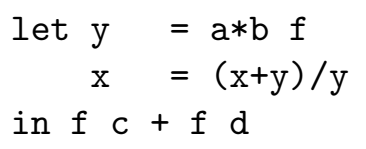

The answer is that Haskell uses a two-dimensional syntax called layout that essentially relies on declarations being "lined up in columns." In the above example, note that y and $f$ begin in the same column. The rules for layout are spelled out in detail in the Report (§2.7, §B.3), but in practice, use of layout is rather intuitive. Just remember two things:

First, the next character following any of the keywords where, let, or of is what determines the starting column for the declarations in the where, let, or case expression being written (the rule also applies to where used in the class and instance declarations to be introduced in Section 5). Thus we can begin the declarations on the same line as the keyword, the next line, etc. (The do keyword, to be discussed later, also uses layout).

Second, just be sure that the starting column is further to the right than the starting column associated with the immediately surrounding clause (otherwise it would be ambiguous). The "termination" of a declaration happens when something appears at or to the left of the starting column associated with that binding form. ${ }^{11}$

Layout is actually shorthand for an explicit grouping mechanism, which deserves mention because it can be useful under certain circumstances. The let example above is equivalent to:

let $\{\mathrm{y}=\mathrm{a} * \mathrm{~b}$

; $\mathrm{f} x=(\mathrm{x}+\mathrm{y}) / \mathrm{y}$

\}

in $f c+f d$

Note the explicit curly braces and semicolons. One way in which this explicit notation is useful is when more than one declaration is desired on a line; for example, this is a valid expression:

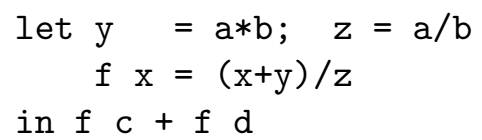

For another example of the expansion of layout into explicit delimiters, see $\S 2.7$.

The use of layout greatly reduces the syntactic clutter associated with declaration lists, thus enhancing readability. It is easy to learn, and its use is encouraged.

\section{$5 \quad$ Type Classes and Overloading}

There is one final feature of Haskell's type system that sets it apart from other programming languages. The kind of polymorphism that we have talked about so far is commonly called parametric polymorphism. There is another kind called ad hoc polymorphism, better known as overloading. Here are some examples of ad hoc polymorphism:

- The literals 1, 2, etc. are often used to represent both fixed and arbitrary precision integers.

- Numeric operators such as + are often defined to work on many different kinds of numbers.

\footnotetext{
${ }^{11}$ Haskell observes the convention that tabs count as 8 blanks; thus care must be taken when using an editor which may observe some other convention.
} 
- The equality operator (== in Haskell) usually works on numbers and many other (but not all) types.

Note that these overloaded behaviors are different for each type (in fact the behavior is sometimes undefined, or error), whereas in parametric polymorphism the type truly does not matter (fringe, for example, really doesn't care what kind of elements are found in the leaves of a tree). In Haskell, type classes provide a structured way to control ad hoc polymorphism, or overloading.

Let's start with a simple, but important, example: equality. There are many types for which we would like equality defined, but some for which we would not. For example, comparing the equality of functions is generally considered computationally intractable, whereas we often want to compare two lists for equality. ${ }^{12}$ To highlight the issue, consider this definition of the function elem which tests for membership in a list:

$$
\begin{array}{ll}
\mathrm{x} \text { 'elem' }[] & =\text { False } \\
\mathrm{x}^{\prime} \text { 'elem' (y:ys) } & =\mathrm{x}==\mathrm{y} \mid \mathrm{l} \text { ( } \mathrm{x} \text { 'elem'ys) }
\end{array}
$$

[For the stylistic reason we discussed in Section 3.1, we have chosen to define elem in infix form. $==$ and || are the infix operators for equality and logical or, respectively.]

Intuitively speaking, the type of elem "ought" to be: a->[a]->Bool. But this would imply that $==$ has type $\mathbf{a}->\mathrm{a}->$ Bool, even though we just said that we don't expect $==$ to be defined for all types.

Furthermore, as we have noted earlier, even if == were defined on all types, comparing two lists for equality is very different from comparing two integers. In this sense, we expect $==$ to be overloaded to carry on these various tasks.

Type classes conveniently solve both of these problems. They allow us to declare which types are instances of which class, and to provide definitions of the overloaded operations associated with a class. For example, let's define a type class containing an equality operator:

class Eq a where

(=) $\quad::$ a $\rightarrow$ a $\rightarrow$ Bool

Here $\mathrm{Eq}$ is the name of the class being defined, and $==$ is the single operation in the class. This declaration may be read "a type a is an instance of the class Eq if there is an (overloaded) operation $==$, of the appropriate type, defined on it." (Note that $==$ is only defined on pairs of objects of the same type.)

The constraint that a type a must be an instance of the class Eq is written Eq a. Thus Eq a is not a type expression, but rather it expresses a constraint on a type, and is called a context. Contexts are placed at the front of type expressions. For example, the effect of the above class declaration is to assign the following type to $==$ :

$$
::(\text { Eq a) } \Rightarrow \text { a } \rightarrow \text { a } \rightarrow \text { Bool }
$$

This should be read, "For every type a that is an instance of the class Eq, == has type a->a->Bool". This is the type that would be used for $==$ in the elem example, and indeed the constraint imposed by the context propagates to the principal type for elem:

elem

$$
::(\text { Eq a) } \Rightarrow \text { a } \rightarrow[a] \rightarrow \text { Bool }
$$

This is read, "For every type a that is an instance of the class Eq, elem has type a->[a]->Bool". This is just what we want - it expresses the fact that elem is not defined on all types, just those for which we know how to compare elements for equality.

\footnotetext{
${ }^{12}$ The kind of equality we are referring to here is "value equality," and opposed to the "pointer equality" found, for example, with Java's ==. Pointer equality is not referentially transparent, and thus does not sit well in a purely functional language.
} 
So far so good. But how do we specify which types are instances of the class Eq, and the actual behavior of $==$ on each of those types? This is done with an instance declaration. For example:

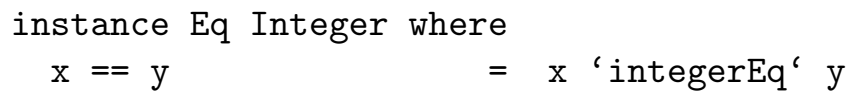

The definition of $==$ is called a method. The function integerEq happens to be the primitive function that compares integers for equality, but in general any valid expression is allowed on the right-hand side, just as for any other function definition. The overall declaration is essentially saying: "The type Integer is an instance of the class Eq, and here is the definition of the method corresponding to the operation ==." Given this declaration, we can now compare fixed precision integers for equality using $==$. Similarly:

instance Eq Float where

$\mathrm{x}==\mathrm{y} \quad=\mathrm{x}$ 'floatEq' $\mathrm{y}$

allows us to compare floating point numbers using $==$.

Recursive types such as Tree defined earlier can also be handled:

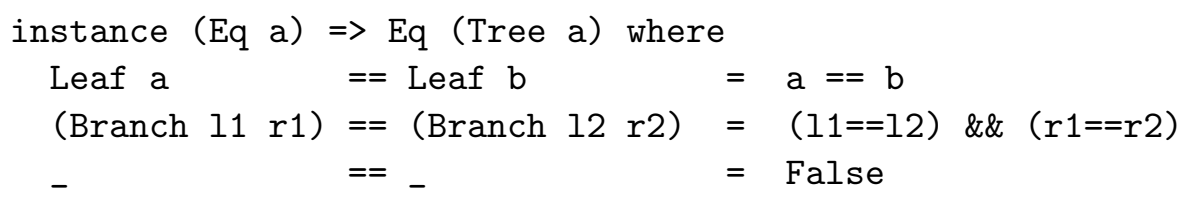

Note the context Eq a in the first line - this is necessary because the elements in the leaves (of type a) are compared for equality in the second line. The additional constraint is essentially saying that we can compare trees of a's for equality as long as we know how to compare a's for equality. If the context were omitted from the instance declaration, a static type error would result.

The Haskell Report, especially the Prelude, contains a wealth of useful examples of type classes. Indeed, a class Eq is defined that is slightly larger than the one defined earlier:

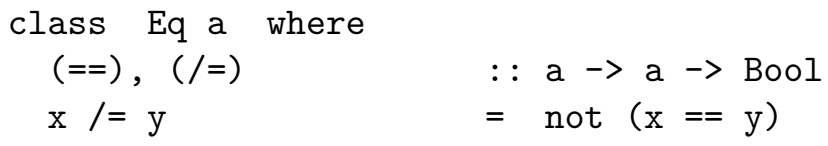

This is an example of a class with two operations, one for equality, the other for inequality. It also demonstrates the use of a default method, in this case for the inequality operation /=. If a method for a particular operation is omitted in an instance declaration, then the default one defined in the class declaration, if it exists, is used instead. For example, the three instances of Eq defined earlier will work perfectly well with the above class declaration, yielding just the right definition of inequality that we want: the logical negation of equality.

Haskell also supports a notion of class extension. For example, we may wish to define a class Ord which inherits all of the operations in Eq, but in addition has a set of comparison operations and minimum and maximum functions:

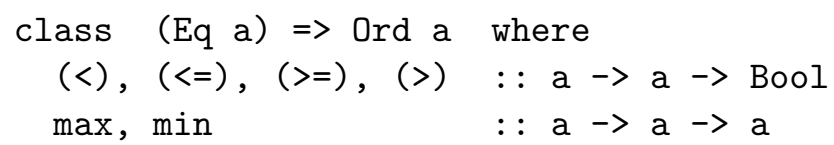

Note the context in the class declaration. We say that Eq is a superclass of Ord (conversely, Ord is a subclass of Eq), and any type which is an instance of Ord must also be an instance of Eq. (In the next Section we give a fuller definition of Ord taken from the Prelude.)

One benefit of such class inclusions is shorter contexts: a type expression for a function that uses operations from both the Eq and Ord classes can use the context (Ord a), rather than 
(Eq a, Ord a), since Ord "implies" Eq. More importantly, methods for subclass operations can assume the existence of methods for superclass operations. For example, the Ord declaration in the Standard Prelude contains this default method for $(<)$ :

$$
\mathrm{x}<\mathrm{y} \quad=\mathrm{x}<=\mathrm{y} \& \& \mathrm{x} /=\mathrm{y}
$$

As an example of the use of Ord, the principal typing of quicksort defined in Section 2.4.1 is:

$$
\text { quicksort } \quad: \text { : }(\text { Ord a) } \Rightarrow[a] \rightarrow[a]
$$

In other words, quicksort only operates on lists of values of ordered types. This typing for quicksort arises because of the use of the comparison operators $<$ and $>=$ in its definition.

Haskell also permits multiple inheritance, since classes may have more than one superclass. For example, the declaration

class (Eq a, Show a) $\Rightarrow C$ a where ...

creates a class $\mathrm{C}$ which inherits operations from both Eq and Show.

Class methods are treated as top level declarations in Haskell. They share the same namespace as ordinary variables; a name cannot be used to denote both a class method and a variable or methods in different classes.

Contexts are also allowed in data declarations; see $\S 4.2 .1$.

Class methods may have additional class constraints on any type variable except the one defining the current class. For example, in this class:

class C a where

$\mathrm{m} \quad:$ : Show $\mathrm{b} \Rightarrow \mathrm{a} \rightarrow \mathrm{b}$

the method $\mathrm{m}$ requires that type $\mathrm{b}$ is in class Show. However, the method $\mathrm{m}$ could not place any additional class constraints on type a. These would instead have to be part of the context in the class declaration.

So far, we have been using "first-order" types. For example, the type constructor Tree has so far always been paired with an argument, as in Tree Integer (a tree containing Integer values) or Tree a (representing the family of trees containing a values). But Tree by itself is a type constructor, and as such takes a type as an argument and returns a type as a result. There are no values in Haskell that have this type, but such "higher-order" types can be used in class declarations.

To begin, consider the following Functor class (taken from the Prelude):

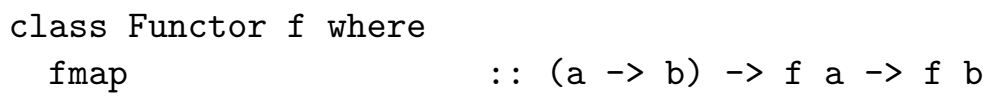

The fmap function generalizes the map function used previously. Note that the type variable $f$ is applied to other types in $f$ a and $f \mathrm{~b}$. Thus we would expect it to be bound to a type such as Tree which can be applied to an argument. An instance of Functor for type Tree would be:

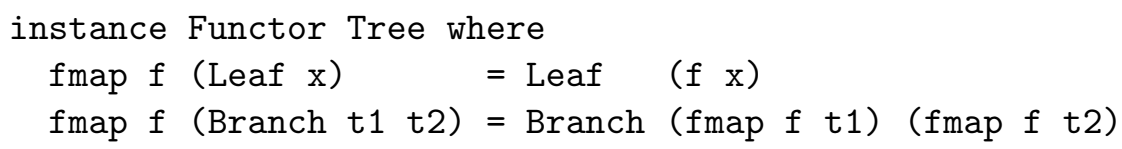

This instance declaration declares that Tree, rather than Tree a, is an instance of Functor. This capability is quite useful, and here demonstrates the ability to describe generic "container" types, allowing functions such as fmap to work uniformly over arbitrary trees, lists, and other data types.

[Type applications are written in the same manner as function applications. The type $\mathrm{T} a \mathrm{~b}$ is parsed as ( $\mathrm{T}$ a) b. Types such as tuples which use special syntax can be written in an 
alternative style which allows currying. For functions, $(->)$ is a type constructor; the types $f \rightarrow g$ and $(->) f g$ are the same. Similarly, the types [a] and [] a are the same. For tuples, the type constructors (as well as the data constructors) are (, ), (, , ), and so on.]

As we know, the type system detects typing errors in expressions. But what about errors due to malformed type expressions? The expression (+) 1233 results in a type error since (+) takes only two arguments. Similarly, the type Tree Int Int should produce some sort of an error since the Tree type takes only a single argument. So, how does Haskell detect malformed type expressions? The answer is a second type system which ensures the correctness of types! Each type has an associated kind which ensures that the type is used correctly.

Type expressions are classified into different kinds which take one of two possible forms:

- The symbol $*$ represents the kind of type associated with concrete data objects. That is, if the value $v$ has type $t$, the kind of $v$ must be $*$.

- If $\kappa_{1}$ and $\kappa_{2}$ are kinds, then $\kappa_{1} \rightarrow \kappa_{2}$ is the kind of types that take a type of kind $\kappa_{1}$ and return a type of kind $\kappa_{2}$.

The type constructor Tree has the kind $* \rightarrow *$; the type Tree Int has the kind $*$. Members of the Functor class must all have the kind $* \rightarrow *$; a kinding error would result from an declaration such as

instance Functor Integer where ...

since Integer has the kind $*$.

Kinds do not appear directly in Haskell programs. The compiler infers kinds before doing type checking without any need for 'kind declarations'. Kinds stay in the background of a Haskell program except when an erroneous type signature leads to a kind error. Kinds are simple enough that compilers should be able to provide descriptive error messages when kind conflicts occur. See $\S 4.1 .1$ and $\S 4.6$ for more information about kinds.

A Different Perspective. Before going on to further examples of the use of type classes, it is worth pointing out two other views of Haskell's type classes. The first is by analogy with object-oriented programming (OOP). In the following general statement about OOP, simply substituting type class for class, and type for object, yields a valid summary of Haskell's type class mechanism:

"Classes capture common sets of operations. A particular object may be an instance of a class, and will have a method corresponding to each operation. Classes may be arranged hierarchically, forming notions of superclasses and subclasses, and permitting inheritance of operations/methods. A default method may also be associated with an operation."

In contrast to OOP, it should be clear that types are not objects, and in particular there is no notion of an object's or type's internal mutable state. An advantage over some OOP languages is that methods in Haskell are completely type-safe: any attempt to apply a method to a value whose type is not in the required class will be detected at compile time instead of at runtime. In other words, methods are not "looked up" at runtime but are simply passed as higher-order functions.

A different perspective can be gotten by considering the relationship between parametric and ad hoc polymorphism. We have shown how parametric polymorphism is useful in defining families of types by universally quantifying over all types. Sometimes, however, that universal quantification is too broad - we wish to quantify over some smaller set of types, such as those types whose elements can be compared for equality. Type classes can be seen as providing a 
structured way to do just this. Indeed, we can think of parametric polymorphism as a kind of overloading too! It's just that the overloading occurs implicitly over all types instead of a constrained set of types (i.e. a type class).

Comparison to Other Languages. The classes used by Haskell are similar to those used in other object-oriented languages such as $\mathrm{C}++$ and Java. However, there are some significant differences:

- Haskell separates the definition of a type from the definition of the methods associated with that type. A class in $\mathrm{C}++$ or Java usually defines both a data structure (the member variables) and the functions associated with the structure (the methods). In Haskell, these definitions are separated.

- The class methods defined by a Haskell class correspond to virtual functions in a $\mathrm{C}++$ class. Each instance of a class provides its own definition for each method; class defaults correspond to default definitions for a virtual function in the base class.

- Haskell classes are roughly similar to a Java interface. Like an interface declaration, a Haskell class declaration defines a protocol for using an object rather than defining an object itself.

- Haskell does not support the $\mathrm{C}++$ overloading style in which functions with different types share a common name.

- The type of a Haskell object cannot be implicitly coerced; there is no universal base class such as Object which values can be projected into or out of.

- $\mathrm{C}++$ and Java attach identifying information (such as a VTable) to the runtime representation of an object. In Haskell, such information is attached logically instead of physically to values, through the type system.

- There is no access control (such as public or private class constituents) built into the Haskell class system. Instead, the module system must be used to hide or reveal components of a class.

\section{Types, Again}

Here we examine some of the more advanced aspects of type declarations.

\subsection{The Newtype Declaration}

A common programming practice is to define a type whose representation is identical to an existing one but which has a separate identity in the type system. In Haskell, the newtype declaration creates a new type from an existing one. For example, natural numbers can be represented by the type Integer using the following declaration:

newtype Natural $=$ MakeNatural Integer

This creates an entirely new type, Natural, whose only constructor contains a single Integer. The constructor MakeNatural converts between an Natural and an Integer: 


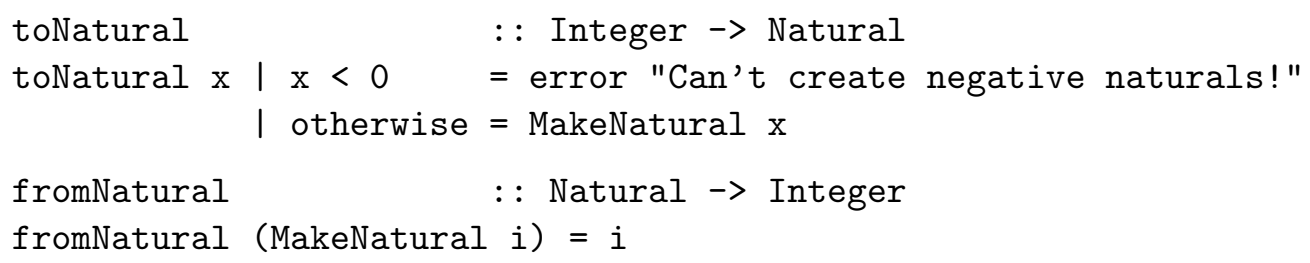

The following instance declaration admits Natural to the Num class:

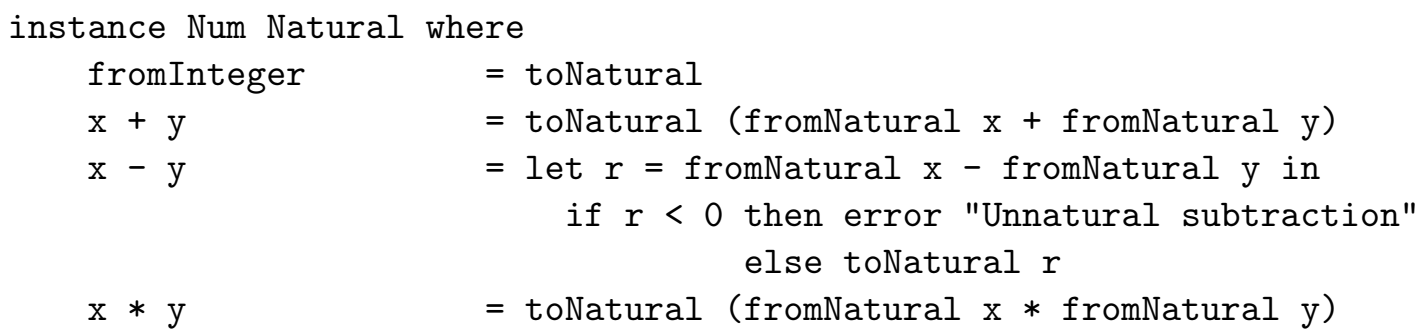

Without this declaration, Natural would not be in Num. Instances declared for the old type do not carry over to the new one. Indeed, the whole purpose of this type is to introduce a different Num instance. This would not be possible if Natural were defined as a type synonym of Integer.

All of this works using a data declaration instead of a newtype declaration. However, the data declaration incurs extra overhead in the representation of Natural values. The use of newtype avoids the extra level of indirection (caused by laziness) that the data declaration would introduce. See section 4.2.3 of the report for a more discussion of the relation between newtype, data, and type declarations.

[Except for the keyword, the newtype declaration uses the same syntax as a data declaration with a single constructor containing a single field. This is appropriate since types defined using newtype are nearly identical to those created by an ordinary data declaration.]

\subsection{Field Labels}

The fields within a Haskell data type can be accessed either positionally or by name using field labels. Consider a data type for a two-dimensional point:

data Point $=$ Pt Float Float

The two components of a Point are the first and second arguments to the constructor Pt. A function such as

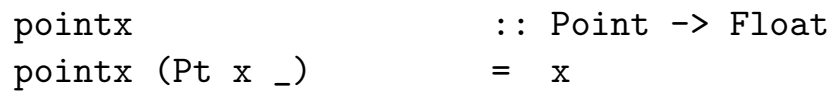

may be used to refer to the first component of a point in a more descriptive way, but, for large structures, it becomes tedious to create such functions by hand.

Constructors in a data declaration may be declared with associated field names, enclosed in braces. These field names identify the components of constructor by name rather than by position. This is an alternative way to define Point:

data Point $=$ Pt $\{$ pointx, pointy : : Float $\}$

This data type is identical to the earlier definition of Point. The constructor Pt is the same in both cases. However, this declaration also defines two field names, pointx and pointy. These field names can be used as selector functions to extract a component from a structure. In this example, the selectors are:

$\begin{array}{lll}\text { pointx } & :: & \text { Point } \rightarrow \text { Float } \\ \text { pointy } & :: & \text { Point } \rightarrow \text { Float }\end{array}$


This is a function using these selectors:

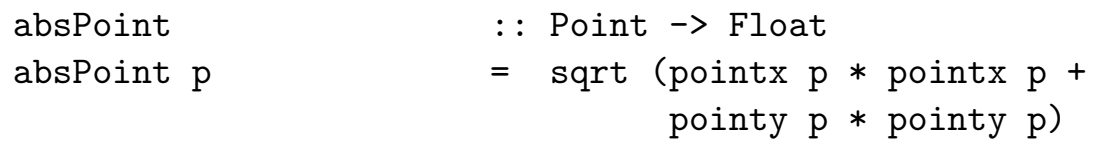

Field labels can also be used to construct new values. The expression Pt \{pointx=1, pointy=2\} is identical to Pt 12 . The use of field names in the declaration of a data constructor does not preclude the positional style of field access; both Pt \{pointx=1, pointy=2\} and Pt 12 are allowed. When constructing a value using field names, some fields may be omitted; these absent fields are undefined.

Pattern matching using field names uses a similar syntax for the constructor Pt:

absPoint $($ Pt $\{$ point $x=x$, pointy $=y\})=\operatorname{sqrt}(x * x+y * y)$

An update function uses field values in an existing structure to fill in components of a new structure. If $\mathrm{p}$ is a Point, then $\mathrm{p}$ \{point $\mathrm{x}=2$ \} is a point with the same pointy as $\mathrm{p}$ but with pointx replaced by 2 . This is not a destructive update: the update function merely creates a new copy of the object, filling in the specified fields with new values.

[The braces used in conjunction with field labels are somewhat special: Haskell syntax usually allows braces to be omitted using the layout rule (described in Section 4.6). However, the braces associated with field names must be explicit.]

Field names are not restricted to types with a single constructor (commonly called 'record' types). In a type with multiple constructors, selection or update operations using field names may fail at runtime. This is similar to the behavior of the head function when applied to an empty list.

Field labels share the top level namespace with ordinary variables and class methods. A field name cannot be used in more than one data type in scope. However, within a data type, the same field name can be used in more than one of the constructors so long as it has the same typing in all cases. For example, in this data type

data $\mathrm{T}=\mathrm{C} 1$ ff : : Int, $\mathrm{g}::$ Float $\}$

I $\mathrm{C} 2$ ff : : Int, $\mathrm{h}::$ Bool $\}$

the field name $f$ applies to both constructors in $T$. Thus if $x$ is of type $T$, then $x\{f=5\}$ will work for values created by either of the constructors in $\mathrm{T}$.

Field names does not change the basic nature of an algebraic data type; they are simply a convenient syntax for accessing the components of a data structure by name rather than by position. They make constructors with many components more manageable since fields can be added or removed without changing every reference to the constructor. For full details of field labels and their semantics, see Section $§ 4.2 .1$.

\subsection{Strict Data Constructors}

Data structures in Haskell are generally lazy: the components are not evaluated until needed. This permits structures that contain elements which, if evaluated, would lead to an error or fail to terminate. Lazy data structures enhance the expressiveness of Haskell and are an essential aspect of the Haskell programming style.

Internally, each field of a lazy data object is wrapped up in a structure commonly referred to as a thunk that encapsulates the computation defining the field value. This thunk is not entered 
until the value is needed; thunks which contain errors $(\perp)$ do not affect other elements of a data structure. For example, the tuple $\left(\right.$ ' $^{\prime}$ ',$\perp$ ) is a perfectly legal Haskell value. The 'a' may be used without disturbing the other component of the tuple. Most programming languages are strict instead of lazy: that is, all components of a data structure are reduced to values before being placed in the structure.

There are a number of overheads associated with thunks: they take time to construct and evaluate, they occupy space in the heap, and they cause the garbage collector to retain other structures needed for the evaluation of the thunk. To avoid these overheads, strictness flags in data declarations allow specific fields of a constructor to be evaluated immediately, selectively suppressing laziness. A field marked by ! in a data declaration is evaluated when the structure is created instead of delayed in a thunk.

There are a number of situations where it may be appropriate to use strictness flags:

- Structure components that are sure to be evaluated at some point during program execution.

- Structure components that are simple to evaluate and never cause errors.

- Types in which partially undefined values are not meaningful.

For example, the complex number library defines the Complex type as:

data RealFloat a $\Rightarrow$ Complex $a=1 a:+$ !

[note the infix definition of the constructor :+.] This definition marks the two components, the real and imaginary parts, of the complex number as being strict. This is a more compact representation of complex numbers but this comes at the expense of making a complex number with an undefined component, $1:+\perp$ for example, totally undefined $(\perp)$. As there is no real need for partially defined complex numbers, it makes sense to use strictness flags to achieve a more efficient representation.

Strictness flags may be used to address memory leaks: structures retained by the garbage collector but no longer necessary for computation.

The strictness flag, !, can only appear in data declarations. It cannot be used in other type signatures or in any other type definitions. There is no corresponding way to mark function arguments as being strict, although the same effect can be obtained using the seq or $! \$$ functions. See $§ 4.2 .1$ for further details.

It is difficult to present exact guidelines for the use of strictness flags. They should be used with caution: laziness is one of the fundamental properties of Haskell and adding strictness flags may lead to hard to find infinite loops or have other unexpected consequences.

\section{Input/Output}

The I/O system in Haskell is purely functional, yet has all of the expressive power found in conventional programming languages. In imperative languages, programs proceed via actions which examine and modify the current state of the world. Typical actions include reading and setting global variables, writing files, reading input, and opening windows. Such actions are also a part of Haskell but are cleanly separated from the purely functional core of the language.

Haskell's I/O system is built around a somewhat daunting mathematical foundation: the monad. However, understanding of the underlying monad theory is not necessary to program using the I/O system. Rather, monads are a conceptual structure into which I/O happens to 
fit. It is no more necessary to understand monad theory to perform Haskell $\mathrm{I} / \mathrm{O}$ than it is to understand group theory to do simple arithmetic. A detailed explanation of monads is found in Section 9 .

The monadic operators that the I/O system is built upon are also used for other purposes; we will look more deeply into monads later. For now, we will avoid the term monad and concentrate on the use of the I/O system. It's best to think of the I/O monad as simply an abstract data type.

Actions are defined rather than invoked within the expression language of Haskell. Evaluating the definition of an action doesn't actually cause the action to happen. Rather, the invocation of actions takes place outside of the expression evaluation we have considered up to this point.

Actions are either atomic, as defined in system primitives, or are a sequential composition of other actions. The I/O monad contains primitives which build composite actions, a process similar to joining statements in sequential order using ';' in other languages. Thus the monad serves as the glue which binds together the actions in a program.

\subsection{Basic I/O Operations}

Every I/O action returns a value. In the type system, the return value is 'tagged' with I0 type, distinguishing actions from other values. For example, the type of the function getChar is:

getChar :: IO Char

The IO Char indicates that getChar, when invoked, performs some action which returns a character. Actions which return no interesting values use the unit type, (). For example, the putChar function:

putChar : : Char $\rightarrow$ IO ()

takes a character as an argument but returns nothing useful. The unit type is similar to void in other languages.

Actions are sequenced using an operator that has a rather cryptic name: $>>=$ (or 'bind'). Instead of using this operator directly, we choose some syntactic sugar, the do notation, to hide these sequencing operators under a syntax resembling more conventional languages. The do notation can be trivially expanded to $>>=$, as described in $\S 3.14$.

The keyword do introduces a sequence of statements which are executed in order. A statement is either an action, a pattern bound to the result of an action using <-, or a set of local definitions introduced using let. The do notation uses layout in the same manner as let or where so we can omit braces and semicolons with proper indentation. Here is a simple program to read and then print a character:

$$
\begin{aligned}
\text { main } & :: \text { IO }() \\
\text { main } & \text { do } c<- \text { getChar } \\
& \text { putChar } c
\end{aligned}
$$

The use of the name main is important: main is defined to be the entry point of a Haskell program (similar to the main function in C), and must have an IO type, usually IO (). (The name main is special only in the module Main; we will have more to say about modules later.) This program performs two actions in sequence: first it reads in a character, binding the result to the variable $\mathrm{c}$, and then prints the character. Unlike a let expression where variables are scoped over all definitions, the variables defined by <- are only in scope in the following statements.

There is still one missing piece. We can invoke actions and examine their results using do, but how do we return a value from a sequence of actions? For example, consider the ready function that reads a character and returns True if the character was a ' $y$ ': 
ready

ready
$:$ : IO Bool

$=$ do $c<-$ getChar

$\mathrm{c}==$ ' $\mathrm{y}$ ' -- Bad!!!

This doesn't work because the second statement in the 'do' is just a boolean value, not an action. We need to take this boolean and create an action that does nothing but return the boolean as its result. The return function does just that:

return $\quad::$ a $\rightarrow$ IO a

The return function completes the set of sequencing primitives. The last line of ready should read return ( $c==$ ' $y$ ').

We are now ready to look at more complicated I/O functions. First, the function getLine:

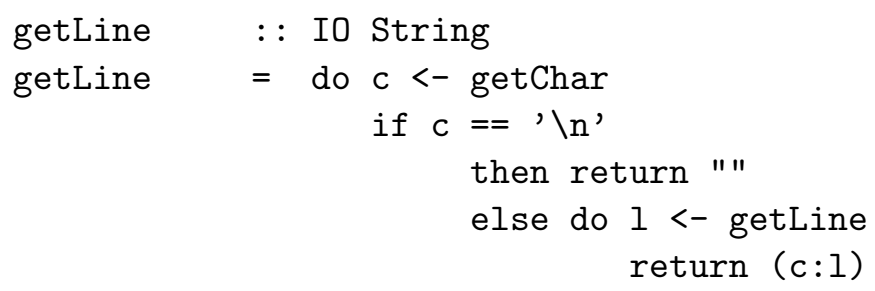

Note the second do in the else clause. Each do introduces a single chain of statements. Any intervening construct, such as the if, must use a new do to initiate further sequences of actions.

The return function admits an ordinary value such as a boolean to the realm of $\mathrm{I} / \mathrm{O}$ actions. What about the other direction? Can we invoke some I/O actions within an ordinary expression? For example, how can we say $\mathrm{x}+$ print $\mathrm{y}$ in an expression so that $\mathrm{y}$ is printed out as the expression evaluates? The answer is that we can't! It is not possible to sneak into the imperative universe while in the midst of purely functional code. Any value 'infected' by the imperative world must be tagged as such. A function such as

$$
\text { f } \quad: \text { : Int } \rightarrow \text { Int } \rightarrow \text { Int }
$$

absolutely cannot do any I/O since IO does not appear in the returned type. This fact is often quite distressing to programmers used to placing print statements liberally throughout their code during debugging. There are, in fact, some unsafe functions available to get around this problem but these are better left to advanced programmers. Debugging packages (like Trace) often make liberal use of these 'forbidden functions' in an entirely safe manner.

\subsection{Programming With Actions}

I/O actions are ordinary Haskell values: they may be passed to functions, placed in structures, and used as any other Haskell value. Consider this list of actions:

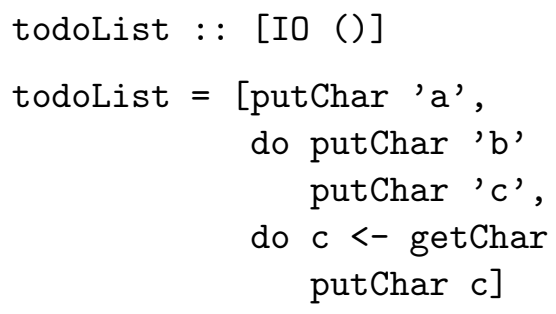

This list doesn't actually invoke any actions - it simply holds them. To join these actions into a single action, a function such as sequence_ is needed:

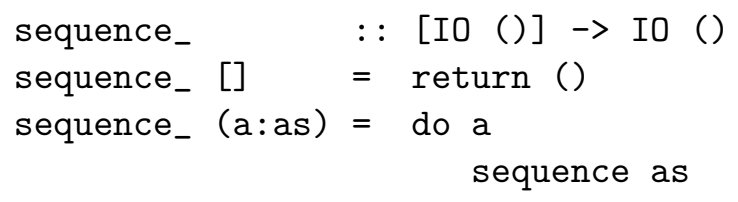


This can be simplified by noting that do $\mathrm{x}$; $\mathrm{y}$ is expanded to $\mathrm{x}>>\mathrm{y}$ (see Section 9.1). This pattern of recursion is captured by the foldr function (see the Prelude for a definition of foldr); a better definition of sequence _ is:

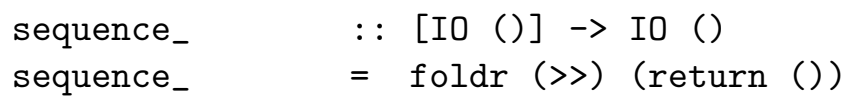

The do notation is a useful tool but in this case the underlying monadic operator, >>, is more appropriate. An understanding of the operators upon which do is built is quite useful to the Haskell programmer.

The sequence_function can be used to construct putStr from putChar:

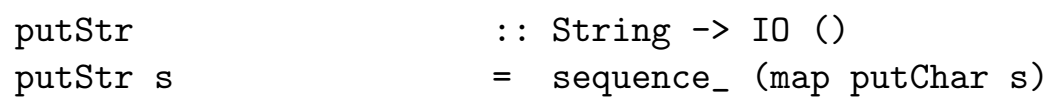

One of the differences between Haskell and conventional imperative programming can be seen in putStr. In an imperative language, mapping an imperative version of putChar over the string would be sufficient to print it. In Haskell, however, the map function does not perform any action. Instead it creates a list of actions, one for each character in the string. The folding operation in sequence_ uses the > function to combine all of the individual actions into a single action. The return () used here is quite necessary - foldr needs a null action at the end of the chain of actions it creates (especially if there are no characters in the string!).

The Prelude and the libraries contains many functions which are useful for sequencing I/O actions. These are usually generalized to arbitrary monads; any function with a context including Monad $\mathrm{m}=>$ works with the IO type.

\subsection{Exception Handling}

So far, we have avoided the issue of exceptions during I/O operations. What would happen if getChar encounters an end of file? ${ }^{13}$ To deal with exceptional conditions such as 'file not found' within the I/O monad, a handling mechanism is used, similar in functionality to the one in standard ML. No special syntax or semantics are used; exception handling is part of the definition of the I/O sequencing operations.

Errors are encoded using a special data type, IOError. This type represents all possible exceptions that may occur within the I/O monad. This is an abstract type: no constructors for IOError are available to the user. Predicates allow IOError values to be queried. For example, the function

isEOFError $\quad:$ : IOError $\rightarrow$ Bool

determines whether an error was caused by an end-of-file condition. By making IOError abstract, new sorts of errors may be added to the system without a noticeable change to the data type. The function isEOFError is defined in a separate library, IO, and must be explicitly imported into a program.

An exception handler has type IOError -> IO a. The catch function associates an exception handler with an action or set of actions:

catch

$$
:: \text { IO a } \rightarrow \text { (IOError } \rightarrow \text { IO a) } \rightarrow \text { IO a }
$$

The arguments to catch are an action and a handler. If the action succeeds, its result is returned without invoking the handler. If an error occurs, it is passed to the handler as a value of type

\footnotetext{
${ }^{13}$ We use the term error for $\perp$ : a condition which cannot be recovered from such as non-termination or pattern match failure. Exceptions, on the other hand, can be caught and handled within the I/O monad.
} 
IOError and the action associated with the handler is then invoked. For example, this version of getChar returns a newline when an error is encountered:

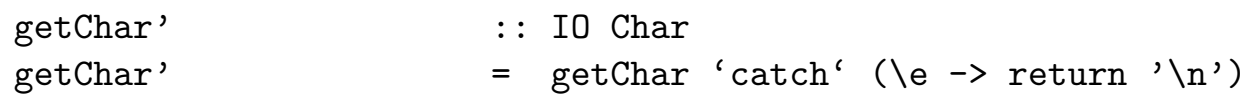

This is rather crude since it treats all errors in the same manner. If only end-of-file is to be recognized, the error value must be queried:

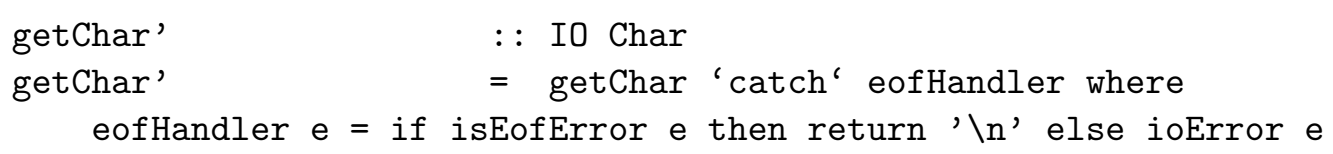

The ioError function used here throws an exception on to the next exception handler. The type of ioError is

ioError

: : IOError $\rightarrow$ IO a

It is similar to return except that it transfers control to the exception handler instead of proceeding to the next I/O action. Nested calls to catch are permitted, and produce nested exception handlers.

Using getChar', we can redefine getLine to demonstrate the use of nested handlers:

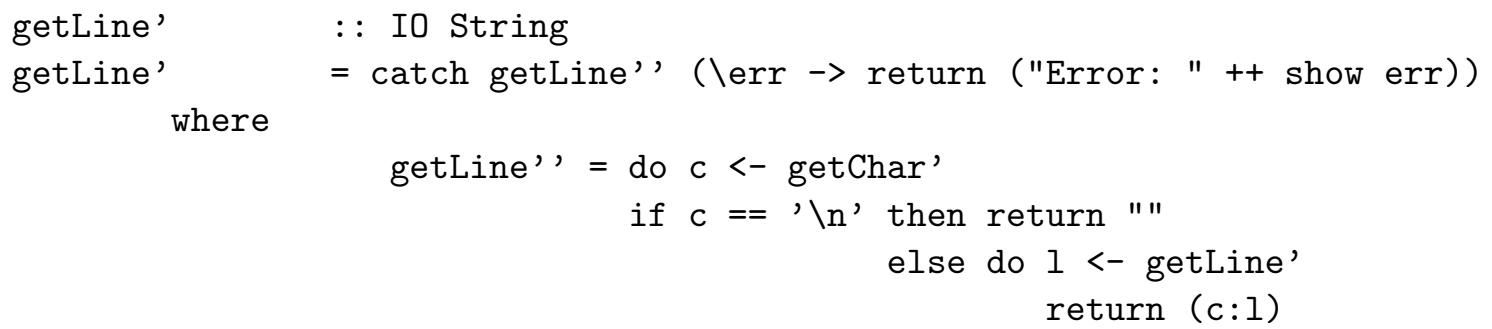

The nested error handlers allow getChar' to catch end of file while any other error results in a string starting with "Error: " from getLine'.

For convenience, Haskell provides a default exception handler at the topmost level of a program that prints out the exception and terminates the program.

\subsection{Files, Channels, and Handles}

Aside from the I/O monad and the exception handling mechanism it provides, I/O facilities in Haskell are for the most part quite similar to those in other languages. Many of these functions are in the IO library instead of the Prelude and thus must be explicitly imported to be in scope (modules and importing are discussed in Section 11). Also, many of these functions are discussed in the Library Report instead of the main report.

Opening a file creates a handle (of type Handle) for use in I/O transactions. Closing the handle closes the associated file:

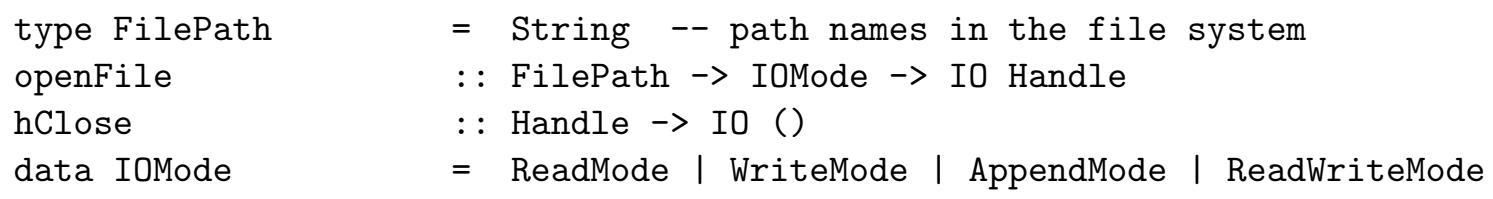

Handles can also be associated with channels: communication ports not directly attached to files. A few channel handles are predefined, including stdin (standard input), stdout (standard output), and stderr (standard error). Character level I/O operations include hGetChar and hPutChar, which take a handle as an argument. The getChar function used previously can be defined as: 
getChar

$=$ hGetChar stdin

Haskell also allows the entire contents of a file or channel to be returned as a single string:

getContents $\quad:$ : Handle $->$ IO String

Pragmatically, it may seem that getContents must immediately read an entire file or channel, resulting in poor space and time performance under certain conditions. However, this is not the case. The key point is that getContents returns a "lazy" (i.e. non-strict) list of characters (recall that strings are just lists of characters in Haskell), whose elements are read "by demand" just like any other list. An implementation can be expected to implement this demand-driven behavior by reading one character at a time from the file as they are required by the computation.

In this example, a Haskell program copies one file to another:

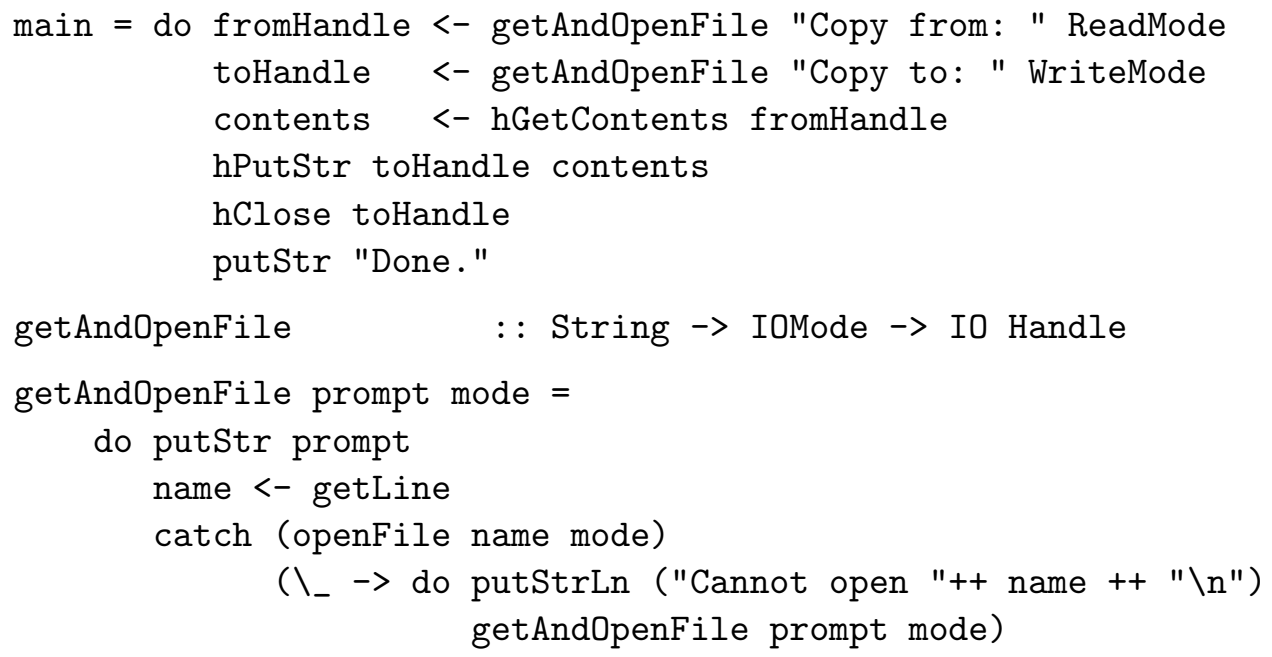

By using the lazy getContents function, the entire contents of the file need not be read into memory all at once. If hPutStr chooses to buffer the output by writing the string in fixed sized blocks of characters, only one block of the input file needs to be in memory at once. The input file is closed implicitly when the last character has been read.

\subsection{Haskell and Imperative Programming}

As a final note, I/O programming raises an important issue: this style looks suspiciously like ordinary imperative programming. For example, the getLine function:

getLine $\quad=$ do $c<-$ getChar

if $c==$ ' $\backslash \mathrm{n}$ '

then return ""

else do $1<-$ getLine

return $(c: 1)$

bears a striking similarity to imperative code (not in any real language) :

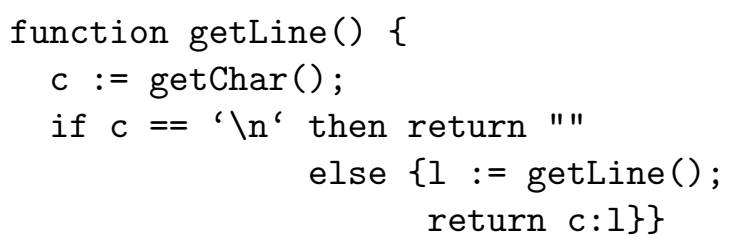

So, in the end, has Haskell simply re-invented the imperative wheel? 
In some sense, yes. The I/O monad constitutes a small imperative sub-language inside Haskell, and thus the I/O component of a program may appear similar to ordinary imperative code. But there is one important difference: There is no special semantics that the user needs to deal with. In particular, equational reasoning in Haskell is not compromised. The imperative feel of the monadic code in a program does not detract from the functional aspect of Haskell. An experienced functional programmer should be able to minimize the imperative component of the program, only using the I/O monad for a minimal amount of top-level sequencing. The monad cleanly separates the functional and imperative program components. In contrast, imperative languages with functional subsets do not generally have any well-defined barrier between the purely functional and imperative worlds.

\section{Standard Haskell Classes}

In this section we introduce the predefined standard type classes in Haskell. We have simplified these classes somewhat by omitting some of the less interesting methods in these classes; the Haskell report contains a more complete description. Also, some of the standard classes are part of the standard Haskell libraries; these are described in the Haskell Library Report.

\subsection{Equality and Ordered Classes}

The classes Eq and Ord have already been discussed. The definition of Ord in the Prelude is somewhat more complex than the simplified version of Ord presented earlier. In particular, note the compare method:

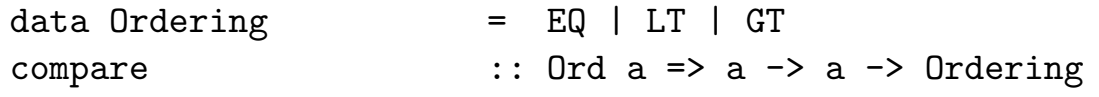

The compare method is sufficient to define all other methods (via defaults) in this class and is the best way to create Ord instances.

\subsection{The Enumeration Class}

Class Enum has a set of operations that underlie the syntactic sugar of arithmetic sequences; for example, the arithmetic sequence expression [1,3..] stands for enumFromThen 13 (see $\S 3.10$ for the formal translation). We can now see that arithmetic sequence expressions can be used to generate lists of any type that is an instance of Enum. This includes not only most numeric types, but also Char, so that, for instance, ['a'..' z'] denotes the list of lower-case letters in alphabetical order. Furthermore, user-defined enumerated types like Color can easily be given Enum instance declarations. If so:

$$
\text { [Red .. Violet] } \Rightarrow \quad \text { [Red, Green, Blue, Indigo, Violet] }
$$

Note that such a sequence is arithmetic in the sense that the increment between values is constant, even though the values are not numbers. Most types in Enum can be mapped onto fixed precision integers; for these, the fromEnum and toEnum convert between Int and a type in Enum.

\subsection{The Read and Show Classes}

The instances of class Show are those types that can be converted to character strings (typically for I/O). The class Read provides operations for parsing character strings to obtain the values they may represent. The simplest function in the class Show is show: 
show

$$
:: \text { (Show a) } \Rightarrow \text { a } \rightarrow \text { String }
$$

Naturally enough, show takes any value of an appropriate type and returns its representation as a character string (list of characters), as in show (2+2), which results in "4". This is fine as far as it goes, but we typically need to produce more complex strings that may have the representations of many values in them, as in

"The sum of " ++ show $\mathrm{x}++$ " and " ++ show $\mathrm{y}++$ " is " ++ show $(\mathrm{x}+\mathrm{y})++$ "."

and after a while, all that concatenation gets to be a bit inefficient. Specifically, let's consider a function to represent the binary trees of Section 2.2.1 as a string, with suitable markings to show the nesting of subtrees and the separation of left and right branches (provided the element type is representable as a string):

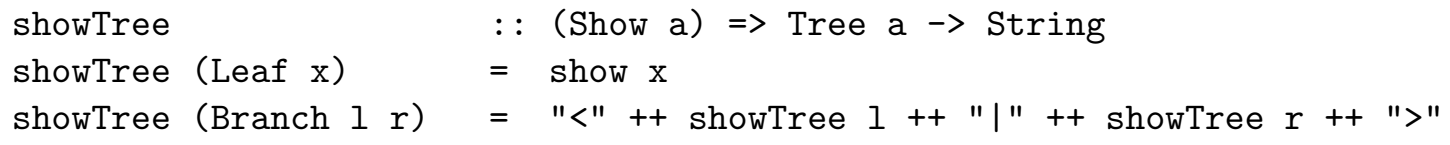

Because (++) has time complexity linear in the length of its left argument, showTree is potentially quadratic in the size of the tree.

To restore linear complexity, the function shows is provided:

shows

$$
::(\text { Show a) } \Rightarrow \text { a } \rightarrow \text { String } \rightarrow \text { String }
$$

shows takes a printable value and a string and returns that string with the value's representation concatenated at the front. The second argument serves as a sort of string accumulator, and show can now be defined as shows with the null accumulator. This is the default definition of show in the Show class definition:

show $\mathrm{x}$ $=\operatorname{shows} \times 1 "$

We can use shows to define a more efficient version of showTree, which also has a string accumulator argument:

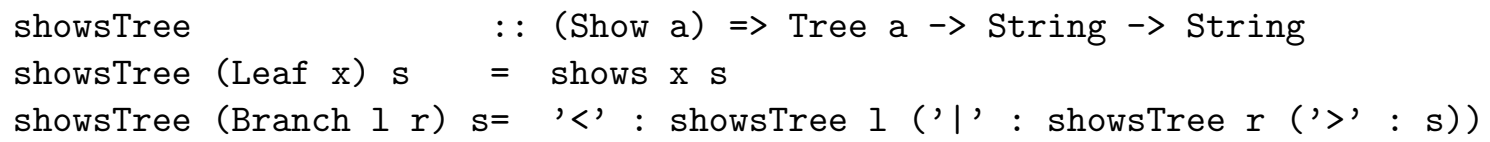

This solves our efficiency problem (showsTree has linear complexity), but the presentation of this function (and others like it) can be improved. First, let's create a type synonym:

type ShowS

$$
=\text { String } \rightarrow \text { String }
$$

This is the type of a function that returns a string representation of something followed by an accumulator string. Second, we can avoid carrying accumulators around, and also avoid amassing parentheses at the right end of long constructions, by using functional composition:

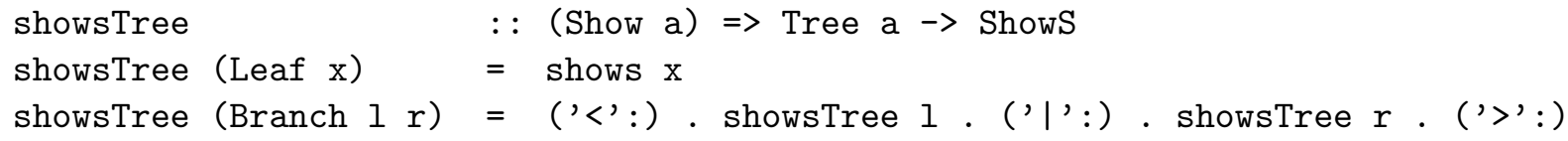

Something more important than just tidying up the code has come about by this transformation: we have raised the presentation from an object level (in this case, strings) to a function level. We can think of the typing as saying that showsTree maps a tree into a showing function. Functions like ('<' :) or ("a string" ++) are primitive showing functions, and we build up more complex functions by function composition.

Now that we can turn trees into strings, let's turn to the inverse problem. The basic idea is a parser for a type a, which is a function that takes a string and returns a list of (a, String) pairs [9]. The Prelude provides a type synonym for such functions:

type ReadS a = String $\rightarrow[$ (a, String $)]$ 
read. We can address both of these problems by using the lexical analyzer provided by the Prelude:

lex

$$
\text { : : ReadS String }
$$

lex normally returns a singleton list containing a pair of strings: the first lexeme in the input string and the remainder of the input. The lexical rules are those of Haskell programs, including comments, which lex skips, along with whitespace. If the input string is empty or contains only whitespace and comments, lex returns [("","" )]; if the input is not empty in this sense, but also does not begin with a valid lexeme after any leading whitespace and comments, lex returns [].

Using the lexical analyzer, our tree parser now looks like this:

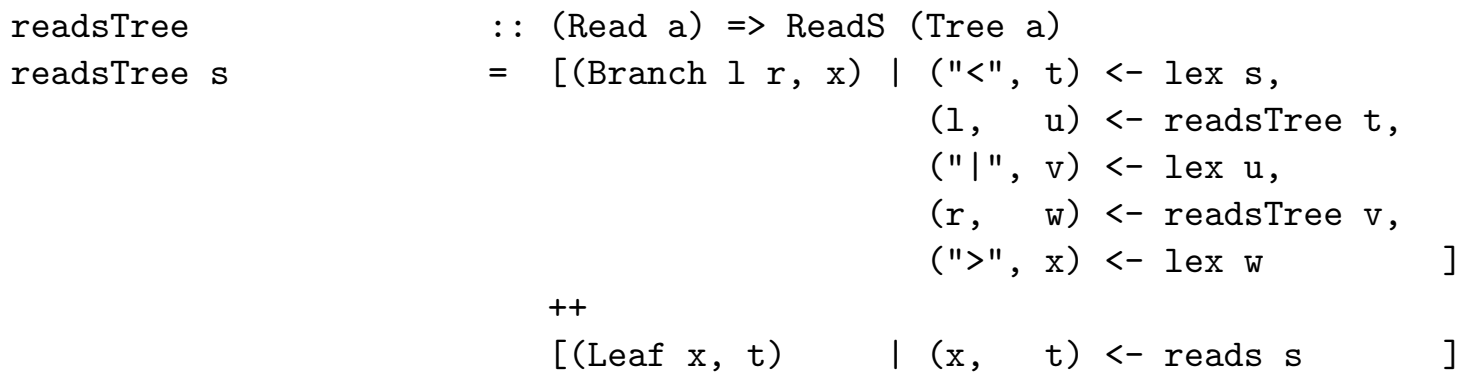

We may now wish to use readsTree and showsTree to declare (Read a) $\Rightarrow$ Tree a an instance of Read and (Show a) $\Rightarrow$ Tree a an instance of Show. This would allow us to use the generic overloaded functions from the Prelude to parse and display trees. Moreover, we would automatically then be able to parse and display many other types containing trees as components, for example, [Tree Integer]. As it turns out, readsTree and showsTree are of almost the right types to be Show and Read methods The showsPrec and readsPrec methods are parameterized versions of shows and reads. The extra parameter is a precedence level, used to properly parenthesize expressions containing infix constructors. For types such as Tree, the precedence can be ignored. The Show and Read instances for Tree are:

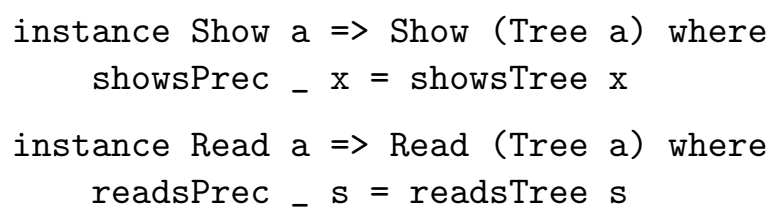

Alternatively, the Show instance could be defined in terms of showTree:

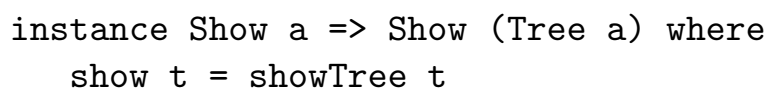

This, however, will be less efficient than the Shows version. Note that the Show class defines default methods for both showsPrec and show, allowing the user to define either one of these in an instance declaration. Since these defaults are mutually recursive, an instance declaration that defines neither of these functions will loop when called. Other classes such as Num also have these "interlocking defaults".

We refer the interested reader to $\S D$ for details of the Read and Show classes.

We can test the Read and Show instances by applying (read . show) (which should be the identity) to some trees, where read is a specialization of reads:

read

$$
::(\text { Read a) } \Rightarrow \text { String } \rightarrow \text { a }
$$

This function fails if there is not a unique parse or if the input contains anything more than a representation of one value of type a (and possibly, comments and whitespace). 


\subsection{Derived Instances}

Recall the Eq instance for trees we presented in Section 5; such a declaration is simple - and boring - to produce: we require that the element type in the leaves be an equality type; then, two leaves are equal iff they contain equal elements, and two branches are equal iff their left and right subtrees are equal, respectively. Any other two trees are unequal:

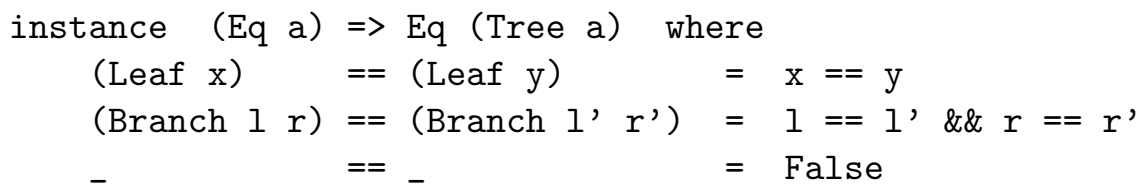

Fortunately, we don't need to go through this tedium every time we need equality operators for a new type; the Eq instance can be derived automatically from the data declaration if we so specify:

data Tree a = Leaf a $\mid$ Branch (Tree a) (Tree a) deriving Eq

The deriving clause implicitly produces an Eq instance declaration just like the one in Section 5. Instances of Ord, Enum, Ix, Read, and Show can also be generated by the deriving clause. [More than one class name can be specified, in which case the list of names must be parenthesized and the names separated by commas.]

The derived Ord instance for Tree is slightly more complicated than the Eq instance:

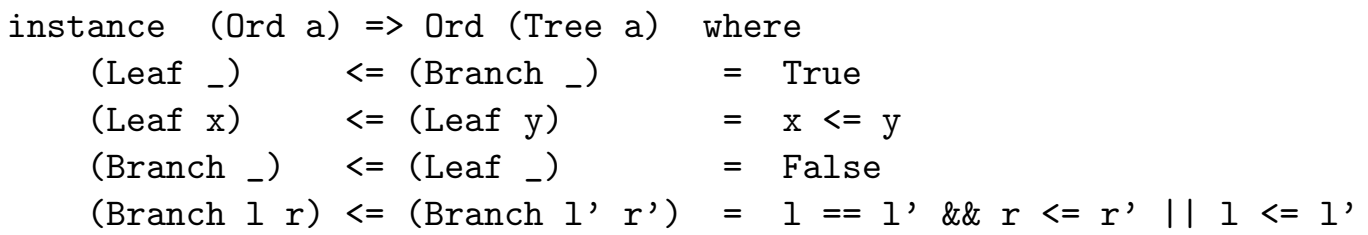

This specifies a lexicographic order: Constructors are ordered by the order of their appearance in the data declaration, and the arguments of a constructor are compared from left to right. Recall that the built-in list type is semantically equivalent to an ordinary two-constructor type. In fact, this is the full declaration:

data $[\mathrm{a}]=[] \mathrm{a}$ a : [a] deriving (Eq, Ord) -- pseudo-code

(Lists also have Show and Read instances, which are not derived.) The derived Eq and Ord instances for lists are the usual ones; in particular, character strings, as lists of characters, are ordered as determined by the underlying Char type, with an initial substring comparing less than a longer string; for example, "cat" < "catalog".

In practice, Eq and Ord instances are almost always derived, rather than user-defined. In fact, we should provide our own definitions of equality and ordering predicates only with some trepidation, being careful to maintain the expected algebraic properties of equivalence relations and total orders. An intransitive (==) predicate, for example, could be disastrous, confusing readers of the program and confounding manual or automatic program transformations that rely on the $(==)$ predicate's being an approximation to definitional equality. Nevertheless, it is sometimes necessary to provide Eq or Ord instances different from those that would be derived; probably the most important example is that of an abstract data type in which different concrete values may represent the same abstract value.

An enumerated type can have a derived Enum instance, and here again, the ordering is that of the constructors in the data declaration. For example:

data Day = Sunday | Monday | Tuesday | Wednesday

| Thursday | Friday | Saturday deriving (Enum) 
Here are some simple examples using the derived instances for this type:

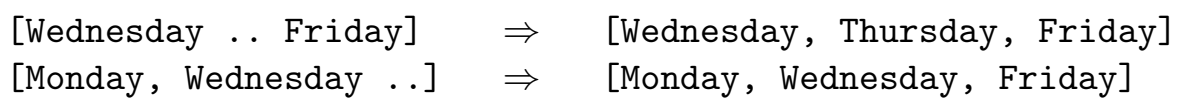

Derived Read (Show) instances are possible for all types whose component types also have Read (Show) instances. (Read and Show instances for most of the standard types are provided by the Prelude. Some types, such as the function type $(->)$, have a Show instance but not a corresponding Read.) The textual representation defined by a derived Show instance is consistent with the appearance of constant Haskell expressions of the type in question. For example, if we add Show and Read to the deriving clause for type Day, above, we obtain

show [Monday .. Wednesday] $\Rightarrow$ "[Monday, Tuesday, Wednesday]"

\section{About Monads}

Many newcomers to Haskell are puzzled by the concept of monads. Monads are frequently encountered in Haskell: the IO system is constructed using a monad, a special syntax for monads has been provided (do expressions), and the standard libraries contain an entire module dedicated to monads. In this section we explore monadic programming in more detail.

This section is perhaps less "gentle" than the others. Here we address not only the language features that involve monads but also try to reveal the bigger picture: why monads are such an important tool and how they are used. There is no single way of explaining monads that works for everyone; more explanations can be found at haskell.org. Another good introduction to practical programming using monads is Wadler's Monads for Functional Programming [10].

\subsection{Monadic Classes}

The Prelude contains a number of classes defining monads are they are used in Haskell. These classes are based on the monad construct in category theory; whilst the category theoretic terminology provides the names for the monadic classes and operations, it is not necessary to delve into abstract mathematics to get an intuitive understanding of how to use the monadic classes.

A monad is constructed on top of a polymorphic type such as IO. The monad itself is defined by instance declarations associating the type with the some or all of the monadic classes, Functor, Monad, and MonadPlus. None of the monadic classes are derivable. In addition to IO, two other types in the Prelude are members of the monadic classes: lists ([]) and Maybe.

Mathematically, monads are governed by set of laws that should hold for the monadic operations. This idea of laws is not unique to monads: Haskell includes other operations that are governed, at least informally, by laws. For example, $\mathrm{x} /=\mathrm{y}$ and not $(\mathrm{x}==\mathrm{y})$ ought to be the same for any type of values being compared. However, there is no guarantee of this: both $==$ and /= are separate methods in the Eq class and there is no way to assure that $==$ and $=/$ are related in this manner. In the same sense, the monadic laws presented here are not enforced by Haskell, but ought be obeyed by any instances of a monadic class. The monad laws give insight into the underlying structure of monads: by examining these laws, we hope to give a feel for how monads are used.

The Functor class, already discussed in section 5, defines a single operation: fmap. The map function applies an operation to the objects inside a container (polymorphic types can be 
thought of as containers for values of another type), returning a container of the same shape. These laws apply to fmap in the class Functor:

$$
\begin{array}{ll}
\text { fmap id } & =\text { id } \\
\text { fmap }(f \cdot g) & =\text { fmap } f \cdot \text { fmap } g
\end{array}
$$

These laws ensure that the container shape is unchanged by fmap and that the contents of the container are not re-arranged by the mapping operation.

The Monad class defines two basic operators: >>= (bind) and return.

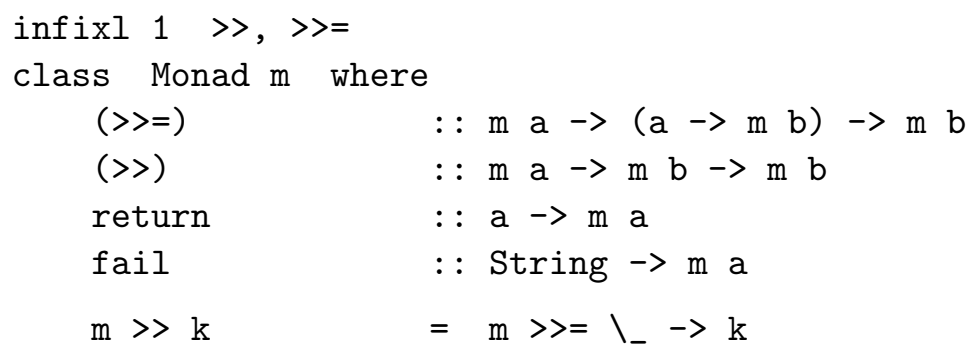

The bind operations, $>>$ and $>>=$, combine two monadic values while the return operation injects a value into the monad (container). The signature of $>>=$ helps us to understand this operation: $\mathrm{ma}>>=\langle\mathrm{v} \rightarrow \mathrm{mb}$ combines a monadic value ma containing values of type a and a function which operates on a value $\mathrm{v}$ of type $\mathrm{a}$, returning the monadic value mb. The result is to combine ma and $\mathrm{mb}$ into a monadic value containing $\mathrm{b}$. The $\gg$ function is used when the function does not need the value produced by the first monadic operator.

The precise meaning of binding depends, of course, on the monad. For example, in the IO monad, $\mathrm{x}>>=\mathrm{y}$ performs two actions sequentially, passing the result of the first into the second. For the other built-in monads, lists and the Maybe type, these monadic operations can be understood in terms of passing zero or more values from one calculation to the next. We will see examples of this shortly.

The do syntax provides a simple shorthand for chains of monadic operations. The essential translation of do is captured in the following two rules:

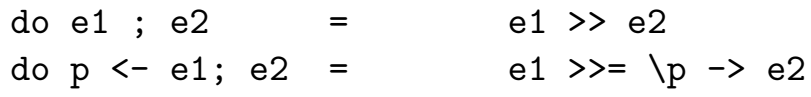

When the pattern in this second form of do is refutable, pattern match failure calls the fail operation. This may raise an error (as in the IO monad) or return a "zero" (as in the list monad). Thus the more complex translation is

$$
\text { do } \mathrm{p}<-\mathrm{e} 1 ; \mathrm{e} 2=\mathrm{e} 1>>=(\backslash \mathrm{v} \rightarrow \text { case } \mathrm{v} \text { of } \mathrm{p} \rightarrow \mathrm{e} 2 ; \text { _ } \rightarrow \text { fail "s") }
$$

where " $\mathrm{s}$ " is a string identifying the location of the do statement for possible use in an error message. For example, in the I/O monad, an action such as 'a' <- getChar will call fail if the character typed is not 'a'. This, in turn, terminates the program since in the I/O monad fail calls error.

The laws which govern $>>=$ and return are:

$$
\begin{aligned}
& \text { return } \mathrm{a}>=\mathrm{k} \quad=\mathrm{k} \mathrm{a} \\
& \mathrm{m} \gg=\text { return } \quad=\mathrm{m} \\
& \mathrm{xs}>>=\text { return. } \mathrm{f} \quad=\text { fmap } \mathrm{f} \mathrm{xs} \\
& \mathrm{m} \gg>=(\backslash \mathrm{x}->\mathrm{k} \mathrm{x}>>=\mathrm{h})=(\mathrm{m}>>=\mathrm{k})>>=\mathrm{h}
\end{aligned}
$$

The class MonadPlus is used for monads that have a zero element and a plus operation: 


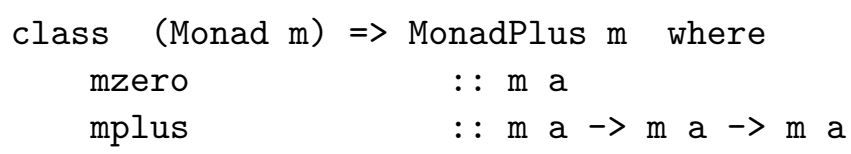

The zero element obeys the following laws:

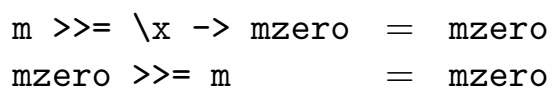

For lists, the zero value is [], the empty list. The I/O monad has no zero element and is not a member of this class.

The laws governing the mplus operator are as follows:

$$
\begin{aligned}
& \mathrm{m} \text { 'mplus' mzero }=\mathrm{m} \\
& \text { mzero 'mplus' } \mathrm{m}=\mathrm{m}
\end{aligned}
$$

The mplus operator is ordinary list concatenation in the list monad.

\subsection{Built-in Monads}

Given the monadic operations and the laws that govern them, what can we build? We have already examined the I/O monad in detail so we start with the two other built-in monads.

For lists, monadic binding involves joining together a set of calculations for each value in the list. When used with lists, the signature of $>>=$ becomes:

$$
(>>=) \quad::[\mathrm{a}] \rightarrow(\mathrm{a}->[\mathrm{b}]) \rightarrow[\mathrm{b}]
$$

That is, given a list of a's and a function that maps an a onto a list of b's, binding applies this function to each of the a's in the input and returns all of the generated b's concatenated into a list. The return function creates a singleton list. These operations should already be familiar: list comprehensions can easily be expressed using the monadic operations defined for lists. These following three expressions are all different syntax for the same thing:

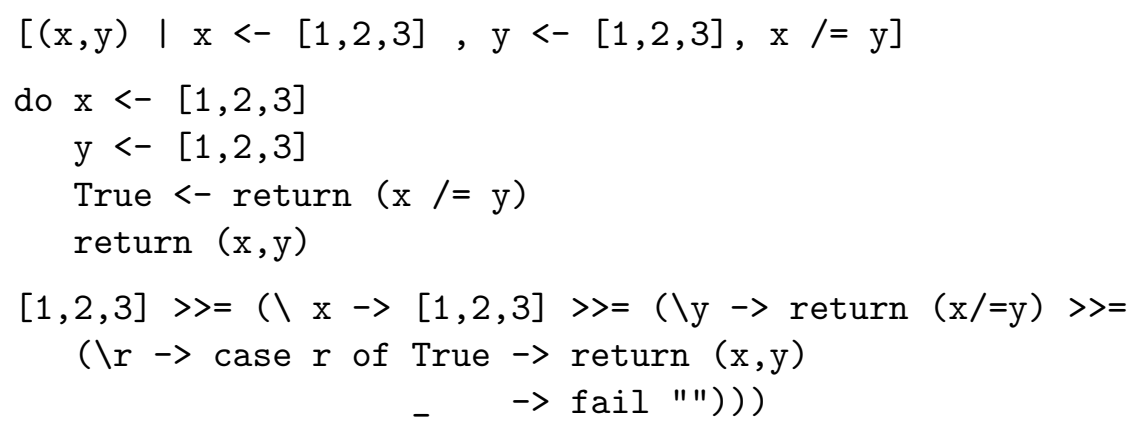

This definition depends on the definition of fail in this monad as the empty list. Essentially, each <- is generating a set of values which is passed on into the remainder of the monadic computation. Thus $\mathrm{x}<-[1,2,3]$ invokes the remainder of the monadic computation three times, once for each element of the list. The returned expression, $(\mathrm{x}, \mathrm{y})$, will be evaluated for all possible combinations of bindings that surround it. In this sense, the list monad can be thought of as describing functions of multi-valued arguments. For example, this function:

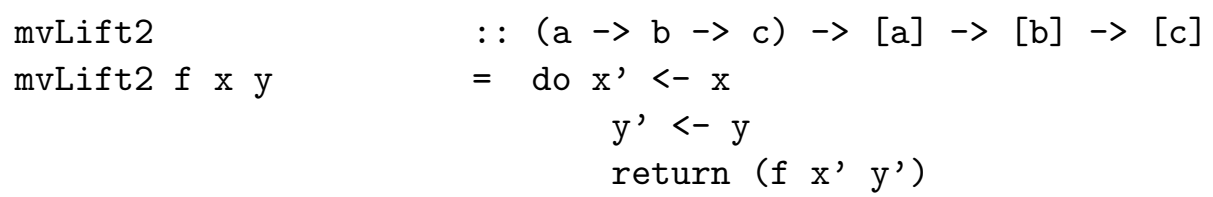

turns an ordinary function of two arguments (f) into a function over multiple values (lists of 
arguments), returning a value for each possible combination of the two input arguments. For example,

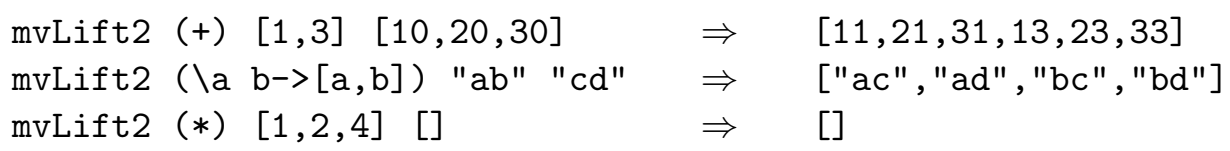

This function is a specialized version of the LiftM2 function in the monad library. You can think of it as transporting a function from outside the list monad, $f$, into the list monad in which computations take on multiple values.

The monad defined for Maybe is similar to the list monad: the value Nothing serves as [] and Just $\mathrm{x}$ as $[\mathrm{x}]$.

\subsection{Using Monads}

Explaining the monadic operators and their associated laws doesn't really show what monads are good for. What they really provide is modularity. That is, by defining an operation monadically, we can hide underlying machinery in a way that allows new features to be incorporated into the monad transparently. Wadler's paper [10] is an excellent example of how monads can be used to construct modular programs. We will start with a monad taken directly from this paper, the state monad, and then build a more complex monad with a similar definition.

Briefly, a state monad built around a state type S looks like this:

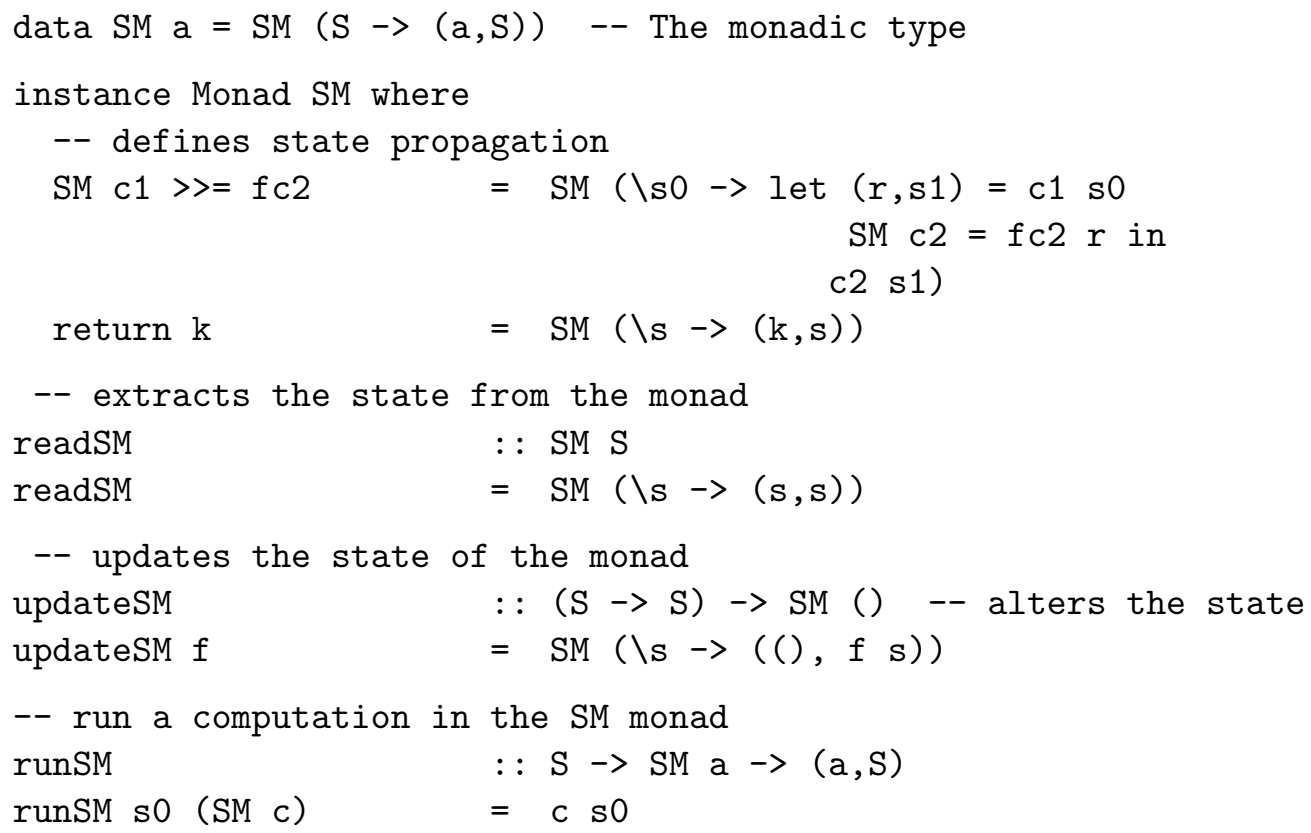

This example defines a new type, SM, to be a computation that implicitly carries a type S. That is, a computation of type SM $t$ defines a value of type $t$ while also interacting with (reading and writing) the state of type S. The definition of SM is simple: it consists of functions that take a state and produce two results: a returned value (of any type) and an updated state. We can't use a type synonym here: we need a type name like SM that can be used in instance declarations. The newtype declaration is often used here instead of data.

This instance declaration defines the 'plumbing' of the monad: how to sequence two computations and the definition of an empty computation. Sequencing (the $>>=$ operator) defines a computation (denoted by the constructor SM) that passes an initial state, s0, into $\mathrm{c} 1$, then passes the value coming out of this computation, $r$, to the function that returns the second 
computation, c2. Finally, the state coming out of c1 is passed into c2 and the overall result is the result of $c 2$.

The definition of return is easier: return doesn't change the state at all; it only serves to bring a value into the monad.

While $>>=$ and return are the basic monadic sequencing operations, we also need some monadic primitives. A monadic primitive is simply an operation that uses the insides of the monad abstraction and taps into the 'wheels and gears' that make the monad work. For example, in the IO monad, operators such as putChar are primitive since they deal with the inner workings of the IO monad. Similarly, our state monad uses two primitives: readSM and updateSM. Note that these depend on the inner structure of the monad - a change to the definition of the SM type would require a change to these primitives.

The definition of readSM and updateSM are simple: readSM brings the state out of the monad for observation while updateSM allows the user to alter the state in the monad. (We could also have used writeSM as a primitive but update is often a more natural way of dealing with state).

Finally, we need a function that runs computations in the monad, runSM. This takes an initial state and a computation and yields both the returned value of the computation and the final state.

Looking at the bigger picture, what we are trying to do is define an overall computation as a series of steps (functions with type SM a), sequenced using >>= and return. These steps may interact with the state (via readSM or updateSM) or may ignore the state. However, the use (or non-use) of the state is hidden: we don't invoke or sequence our computations differently depending on whether or not they use S.

Rather than present any examples using this simple state monad, we proceed on to a more complex example that includes the state monad. We define a small embedded language of resource-using calculations. That is, we build a special purpose language implemented as a set of Haskell types and functions. Such languages use the basic tools of Haskell, functions and types, to build a library of operations and types specifically tailored to a domain of interest.

In this example, consider a computation that requires some sort of resource. If the resource is available, computation proceeds; when the resource is unavailable, the computation suspends. We use the type $\mathrm{R}$ to denote a computation using resources controlled by our monad. The definition of $\mathrm{R}$ is as follows:

data $R$ a $=R$ (Resource $\rightarrow$ (Resource, Either a $(R$ a $)$ ))

Each computation is a function from available resources to remaining resources, coupled with either a result, of type a, or a suspended computation, of type $\mathrm{R}$ a, capturing the work done up to the point where resources were exhausted.

The Monad instance for $\mathrm{R}$ is as follows:

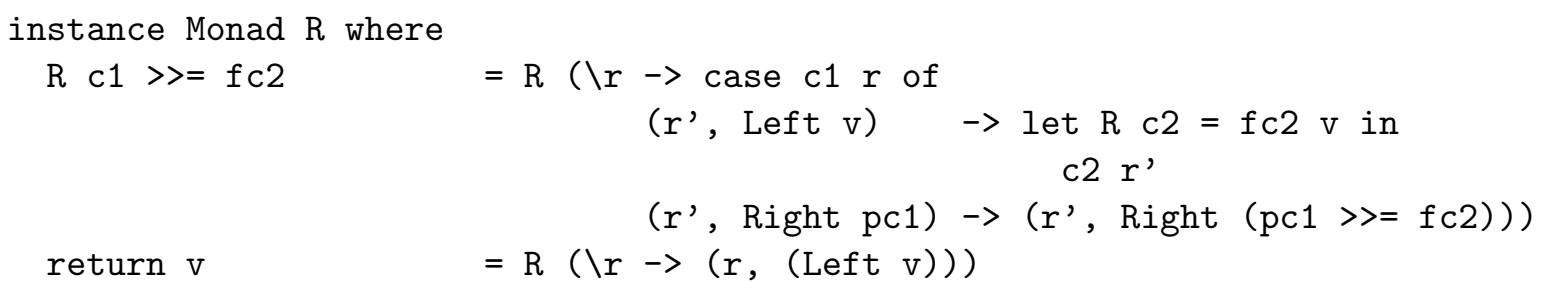

The Resource type is used in the same manner as the state in the state monad. This definition reads as follows: to combine two 'resourceful' computations, $\mathrm{c} 1$ and $\mathrm{fc} 2$ (a function producing c2), pass the initial resources into c1. The result will be either

- a value, $\mathrm{v}$, and remaining resources, which are used to determine the next computation (the call fc2 v), or 
- a suspended computation, pc1, and resources remaining at the point of suspension.

The suspension must take the second computation into consideration: $\mathrm{pc} 1$ suspends only the first computation, $\mathrm{c} 1$, so we must bind $\mathrm{c} 2$ to this to produce a suspension of the overall computation. The definition of return leaves the resources unchanged while moving $\mathrm{v}$ into the monad.

This instance declaration defines the basic structure of the monad but does not determine how resources are used. This monad could be used to control many types of resource or implement many different types of resource usage policies. We will demonstrate a very simple definition of resources as an example: we choose Resource to be an Integer, representing available computation steps:

type Resource = Integer

This function takes a step unless no steps are available:

$$
\begin{aligned}
& \text { step } \quad:: \mathrm{a} \rightarrow \mathrm{R} \text { a } \\
& \text { step } \mathrm{V} \quad=\mathrm{c} \text { where } \\
& c=R(\backslash r \rightarrow \text { if } r /=0 \text { then }(r-1 \text {, Left } v) \\
& \text { else ( } r \text {, Right } c) \text { ) }
\end{aligned}
$$

The Left and Right constructors are part of the Either type. This function continues computation in $\mathrm{R}$ by returning $\mathrm{v}$ so long as there is at least one computational step resource available. If no steps are available, the step function suspends the current computation (this suspension is captured in c) and passes this suspended computation back into the monad.

So far, we have the tools to define a sequence of "resourceful" computations (the monad) and we can express a form of resource usage using step. Finally, we need to address how computations in this monad are expressed.

Consider an increment function in our monad:

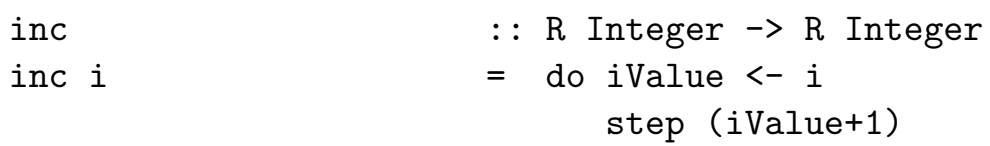

This defines increment as a single step of computation. The <- is necessary to pull the argument value out of the monad; the type of $i$ Value is Integer instead of $R$ Integer.

This definition isn't particularly satisfying, though, compared to the standard definition of the increment function. Can we instead "dress up" existing operations like + so that they work in our monadic world? We'll start with a set of lifting functions. These bring existing functionality into the monad. Consider the definition of lift1 (this is slightly different from the liftM1 found in the Monad library):

lift1

lift1 $f$

$$
\begin{aligned}
& ::(\mathrm{a} \rightarrow \mathrm{b}) \rightarrow(\mathrm{R} \text { a } \rightarrow \mathrm{R} \text { b) } \\
& =\mid r a 1->\text { do a1 <- ra1 } \\
& \text { step ( } f \text { a1) }
\end{aligned}
$$

This takes a function of a single argument, $f$, and creates a function in $R$ that executes the lifted function in a single step. Using lift1, inc becomes

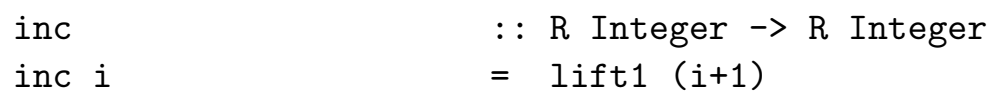

This is better but still not ideal. First, we add lift2:

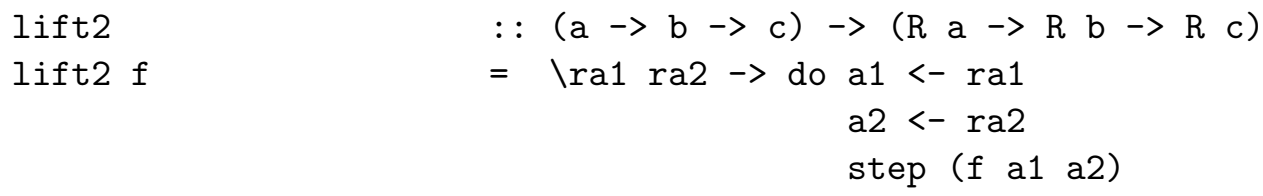


Notice that this function explicitly sets the order of evaluation in the lifted function: the computation yielding a1 occurs before the computation for a2.

Using lift2, we can create a new version of $==$ in the $R$ monad:

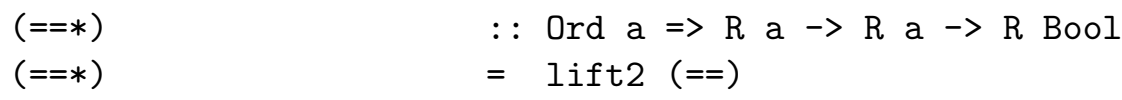

We had to use a slightly different name for this new function since $==$ is already taken but in some cases we can use the same name for the lifted and unlifted function. This instance declaration allows all of the operators in Num to be used in R:

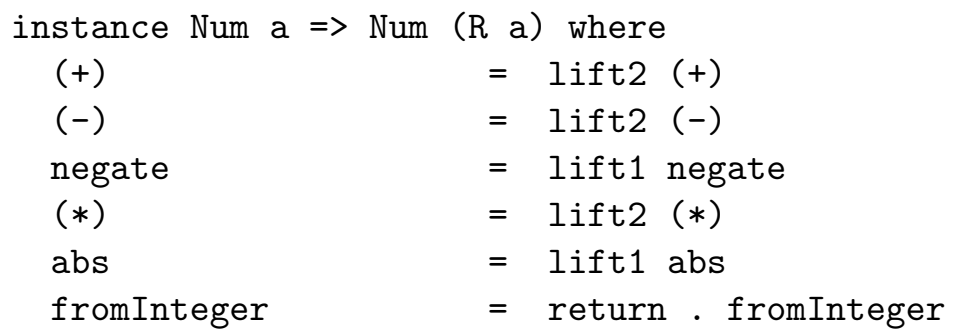

The fromInteger function is applied implicitly to all integer constants in a Haskell program (see Section 10.3); this definition allows integer constants to have the type $\mathrm{R}$ Integer. We can now, finally, write increment in a completely natural style:

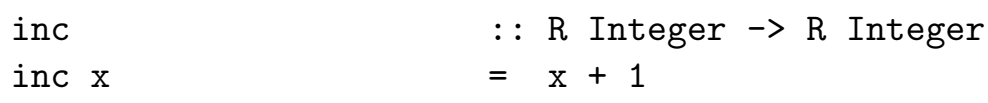

Note that we cannot lift the Eq class in the same manner as the Num class: the signature of $==*$ is not compatible with allowable overloadings of $==$ since the result of $==*$ is $\mathrm{R}$ Bool instead of Bool.

To express interesting computations in R we will need a conditional. Since we can't use if (it requires that the test be of type Bool instead of $R$ Bool), we name the function ifR:

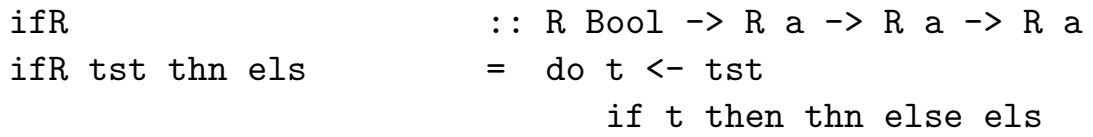

Now we're ready for a larger program in the $\mathrm{R}$ monad:

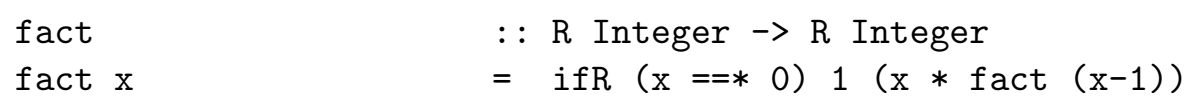

Now this isn't quite the same as an ordinary factorial function but still quite readable. The idea of providing new definitions for existing operations like + or if is an essential part of creating an embedded language in Haskell. Monads are particularly useful for encapsulating the semantics of these embedded languages in a clean and modular way.

We're now ready to actually run some programs. This function runs a program in $M$ given a maximum number of computation steps:

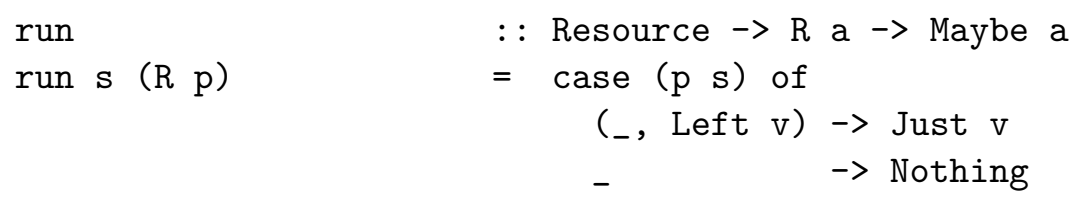

We use the Maybe type to deal with the possibility of the computation not finishing in the allotted number of steps. We can now compute

$$
\begin{array}{lll}
\operatorname{run} 10(\text { fact } 2) & \Rightarrow & \text { Just } 2 \\
\operatorname{run} 10(\text { fact } 20) & \Rightarrow & \text { Nothing }
\end{array}
$$


Finally, we can add some more interesting functionality to this monad. Consider the following function:

$$
:: \mathrm{R} \text { a } \rightarrow \mathrm{R} \text { a } \rightarrow \mathrm{R} \text { a }
$$

This runs two computations in parallel, returning the value of the first one to complete. One possible definition of this function is:

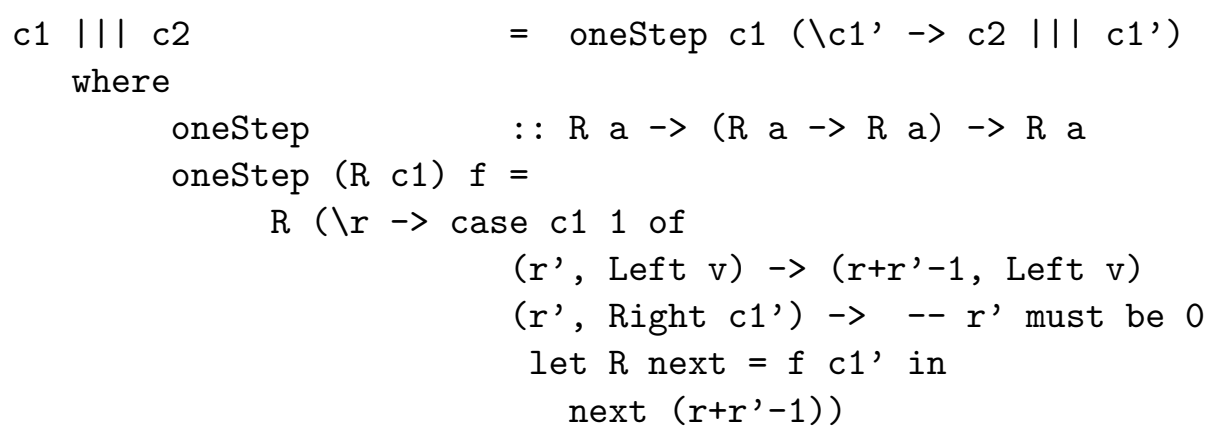

This takes a step in $c 1$, returning its value of $c 1$ complete or, if $c 1$ returns a suspended computation (c1'), it evaluates c2 |II c1'. The oneStep function takes a single step in its argument, either returning an evaluated value or passing the remainder of the computation into $f$. The definition of oneStep is simple: it gives c1 a 1 as its resource argument. If a final value is reached, this is returned, adjusting the returned step count (it is possible that a computation might return after taking no steps so the returned resource count isn't necessarily 0 ). If the computation suspends, a patched up resource count is passed to the final continuation.

We can now evaluate expressions like run 100 (fact (-1) III (fact 3)) without looping since the two calculations are interleaved. (Our definition of fact loops for -1 ). Many variations are possible on this basic structure. For example, we could extend the state to include a trace of the computation steps. We could also embed this monad inside the standard IO monad, allowing computations in $\mathrm{M}$ to interact with the outside world.

While this example is perhaps more advanced than others in this tutorial, it serves to illustrate the power of monads as a tool for defining the basic semantics of a system. We also present this example as a model of a small Domain Specific Language, something Haskell is particularly good at defining. Many other DSLs have been developed in Haskell; see haskell.org for many more examples. Of particular interest are Fran, a language of reactive animations, and Haskore, a language of computer music.

\section{Numbers}

Haskell provides a rich collection of numeric types, based on those of Scheme [7], which in turn are based on Common Lisp [8]. (Those languages, however, are dynamically typed.) The standard types include fixed- and arbitrary-precision integers, ratios (rational numbers) formed from each integer type, and single- and double-precision real and complex floating-point. We outline here the basic characteristics of the numeric type class structure and refer the reader to $\S 6.4$ for details.

\subsection{Numeric Class Structure}

The numeric type classes (class Num and those that lie below it) account for many of the standard Haskell classes. We also note that Num is a subclass of Eq, but not of Ord; this is because the order predicates do not apply to complex numbers. The subclass Real of Num, however, is a subclass of Ord as well. 
The Num class provides several basic operations common to all numeric types; these include, among others, addition, subtraction, negation, multiplication, and absolute value:

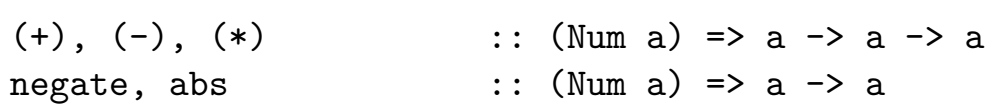

[negate is the function applied by Haskell's only prefix operator, minus; we can't call it (-), because that is the subtraction function, so this name is provided instead. For example, $-\mathrm{x} * \mathrm{y}$ is equivalent to negate $(\mathrm{x} * \mathrm{y})$. (Prefix minus has the same syntactic precedence as infix minus, which, of course, is lower than that of multiplication.)]

Note that Num does not provide a division operator; two different kinds of division operators are provided in two non-overlapping subclasses of Num:

The class Integral provides whole-number division and remainder operations. The standard instances of Integral are Integer (unbounded or mathematical integers, also known as "bignums") and Int (bounded, machine integers, with a range equivalent to at least 29-bit signed binary). A particular Haskell implementation might provide other integral types in addition to these. Note that Integral is a subclass of Real, rather than of Num directly; this means that there is no attempt to provide Gaussian integers.

All other numeric types fall in the class Fractional, which provides the ordinary division operator $(/)$. The further subclass Floating contains trigonometric, logarithmic, and exponential functions.

The RealFrac subclass of Fractional and Real provides a function properFraction, which decomposes a number into its whole and fractional parts, and a collection of functions that round to integral values by differing rules:

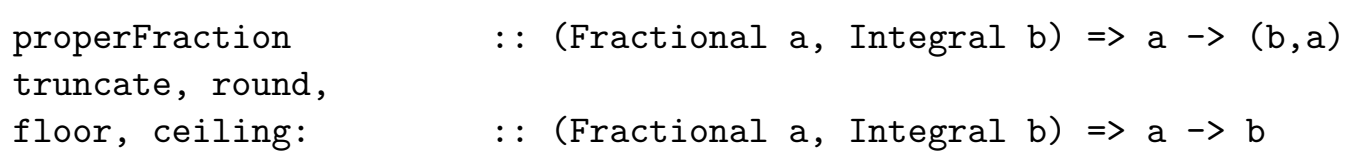

The RealFloat subclass of Floating and RealFrac provides some specialized functions for efficient access to the components of a floating-point number, the exponent and significand. The standard types Float and Double fall in class RealFloat.

\subsection{Constructed Numbers}

Of the standard numeric types, Int, Integer, Float, and Double are primitive. The others are made from these by type constructors.

Complex (found in the library Complex) is a type constructor that makes a complex type in class Floating from a RealFloat type:

data (Realfloat a) $\Rightarrow$ Complex $a=$ ! a $:+$ ! a deriving (Eq, Text)

The ! symbols are strictness flags; these were discussed in Section 6.3. Notice the context RealFloat a, which restricts the argument type; thus, the standard complex types are Complex Float and Complex Double. We can also see from the data declaration that a complex number is written $x:+y$; the arguments are the cartesian real and imaginary parts, respectively. Since $:+$ is a data constructor, we can use it in pattern matching:

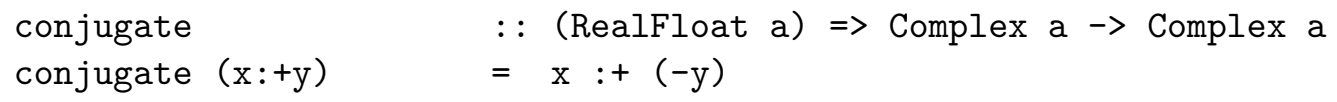

Similarly, the type constructor Ratio (found in the Rational library) makes a rational type in class RealFrac from an instance of Integral. (Rational is a type synonym for 


\subsection{Default Numeric Types}

Consider the following function definition:

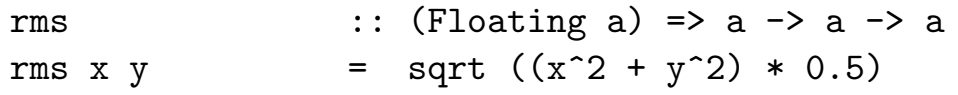

The exponentiation function $\left({ }^{\wedge}\right)$ (one of three different standard exponentiation operators with different typings, see $\S 6.8 .5$ ) has the type (Num a, Integral $\mathrm{b}$ ) $\Rightarrow \mathrm{a} \rightarrow \mathrm{b} \rightarrow \mathrm{a}$, and since 2 has the type $\left(\right.$ Num a) $\Rightarrow>$ a, the type of $x^{\wedge} 2$ is (Num a, Integral b) $\Rightarrow$ a. This is a problem; there is no way to resolve the overloading associated with the type variable $b$, since it is in the context, but has otherwise vanished from the type expression. Essentially, the programmer has specified that $\mathrm{x}$ should be squared, but has not specified whether it should be squared with an Int or an Integer value of two. Of course, we can fix this:

$\operatorname{rms} \mathrm{x} y=\operatorname{sqrt}\left(\left(\mathrm{x}^{-}(2:\right.\right.$ : Integer $)+\mathrm{y}^{-}(2::$ Integer $\left.\left.)\right) * 0.5\right)$

It's obvious that this sort of thing will soon grow tiresome, however.

In fact, this kind of overloading ambiguity is not restricted to numbers:

show (read "xyz")

As what type is the string supposed to be read? This is more serious than the exponentiation ambiguity, because there, any Integral instance will do, whereas here, very different behavior can be expected depending on what instance of Text is used to resolve the ambiguity.

Because of the difference between the numeric and general cases of the overloading ambiguity problem, Haskell provides a solution that is restricted to numbers: Each module may contain a default declaration, consisting of the keyword default followed by a parenthesized, commaseparated list of numeric monotypes (types with no variables). When an ambiguous type variable is discovered (such as b, above), if at least one of its classes is numeric and all of its classes are standard, the default list is consulted, and the first type from the list that will satisfy the context of the type variable is used. For example, if the default declaration default (Int, Float) is in effect, the ambiguous exponent above will be resolved as type Int. (See §4.3.4 for more details.)

The "default default" is (Integer, Double), but (Integer, Rational, Double) may also be appropriate. Very cautious programmers may prefer default (), which provides no defaults.

\section{Modules}

A Haskell program consists of a collection of modules. A module in Haskell serves the dual purpose of controlling name-spaces and creating abstract data types.

The top level of a module contains any of the various declarations we have discussed: fixity declarations, data and type declarations, class and instance declarations, type signatures, function definitions, and pattern bindings. Except for the fact that import declarations (to be described shortly) must appear first, the declarations may appear in any order (the top-level scope is mutually recursive).

Haskell's module design is relatively conservative: the name-space of modules is completely flat, and modules are in no way "first-class." Module names are alphanumeric and must begin with an uppercase letter. There is no formal connection between a Haskell module and the file system that would (typically) support it. In particular, there is no connection between module names and file names, and more than one module could conceivably reside in a single file (one module may even span several files). Of course, a particular implementation will most likely adopt conventions that make the connection between modules and files more stringent. 
Technically speaking, a module is really just one big declaration which begins with the keyword module; here's an example for a module whose name is Tree:

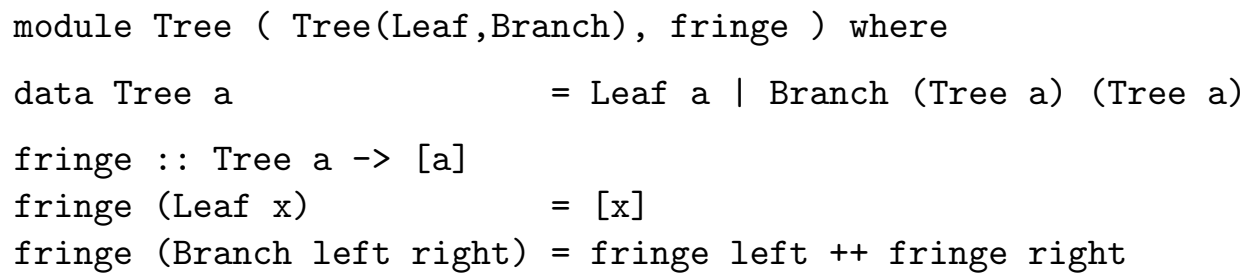

The type Tree and the function fringe should be familiar; they were given as examples in Section 2.2.1. [Because of the where keyword, layout is active at the top level of a module, and thus the declarations must all line up in the same column (typically the first). Also note that the module name is the same as that of the type; this is allowed.]

This module explicitly exports Tree, Leaf, Branch, and fringe. If the export list following the module keyword is omitted, all of the names bound at the top level of the module would be exported. (In the above example everything is explicitly exported, so the effect would be the same.) Note that the name of a type and its constructors have be grouped together, as in Tree (Leaf, Branch). As short-hand, we could also write Tree(...). Exporting a subset of the constructors is also possible. The names in an export list need not be local to the exporting module; any name in scope may be listed in an export list.

The Tree module may now be imported into some other module:

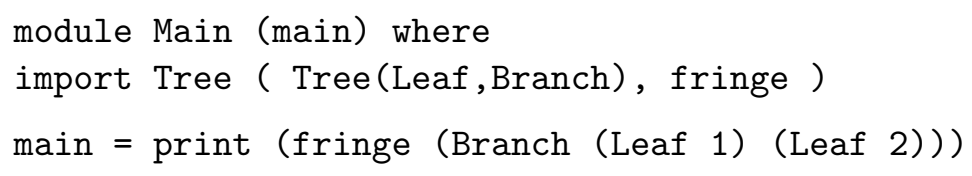

The various items being imported into and exported out of a module are called entities. Note the explicit import list in the import declaration; omitting it would cause all entities exported from Tree to be imported.

\subsection{Qualified Names}

There is an obvious problem with importing names directly into the namespace of module. What if two imported modules contain different entities with the same name? Haskell solves this problem using qualified names. An import declaration may use the qualified keyword to cause the imported names to be prefixed by the name of the module imported. These prefixes are followed by the '.' character without intervening whitespace. [Qualifiers are part of the lexical syntax. Thus, A.x and A . $\mathrm{x}$ are quite different: the first is a qualified name and the second a use of the infix '.' function.] For example, using the Tree module introduced above:

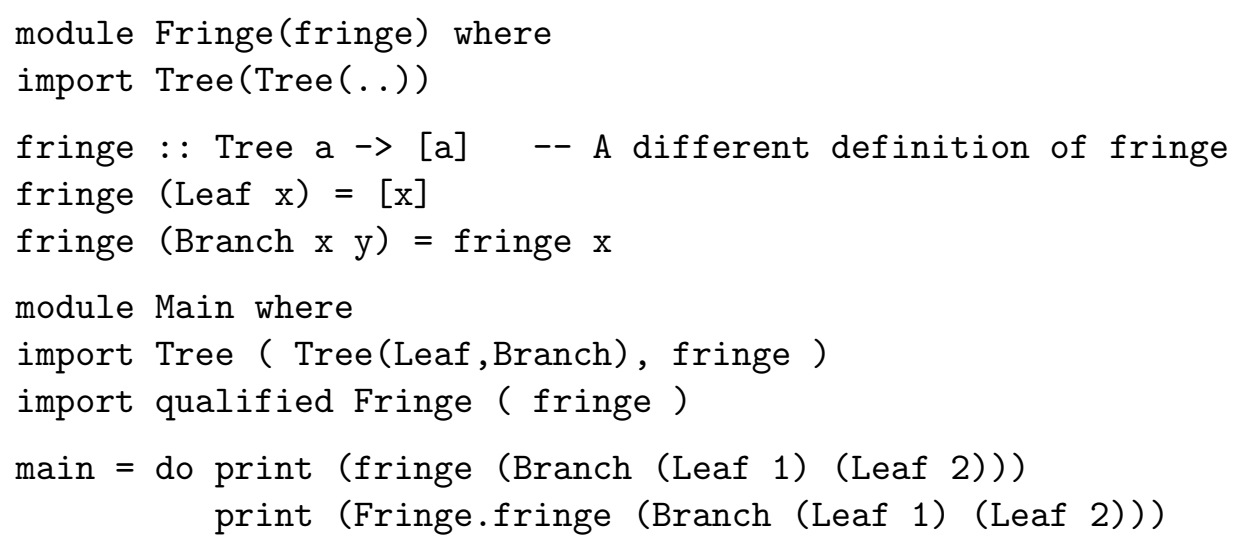

Some Haskell programmers prefer to use qualifiers for all imported entities, making the source 
of each name explicit with every use. Others prefer short names and only use qualifiers when absolutely necessary.

Qualifiers are used to resolve conflicts between different entities which have the same name. But what if the same entity is imported from more than one module? Fortunately, such name clashes are allowed: an entity can be imported by various routes without conflict. The compiler knows whether entities from different modules are actually the same.

\subsection{Abstract Data Types}

Aside from controlling namespaces, modules provide the only way to build abstract data types (ADTs) in Haskell. For example, the characteristic feature of an ADT is that the representation type is hidden; all operations on the ADT are done at an abstract level which does not depend on the representation. For example, although the Tree type is simple enough that we might not normally make it abstract, a suitable ADT for it might include the following operations:

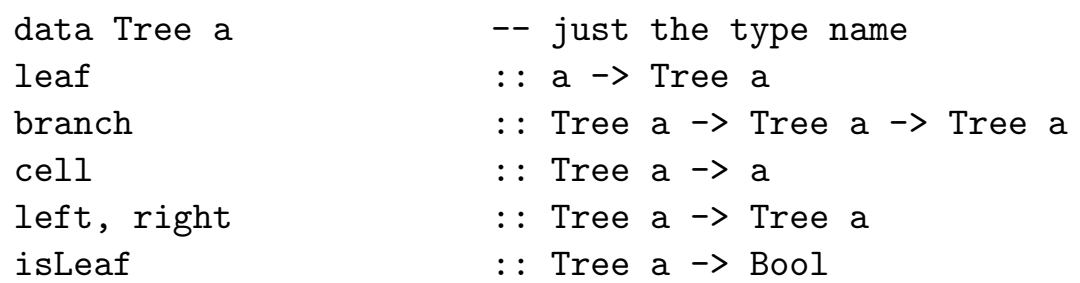

A module supporting this is:

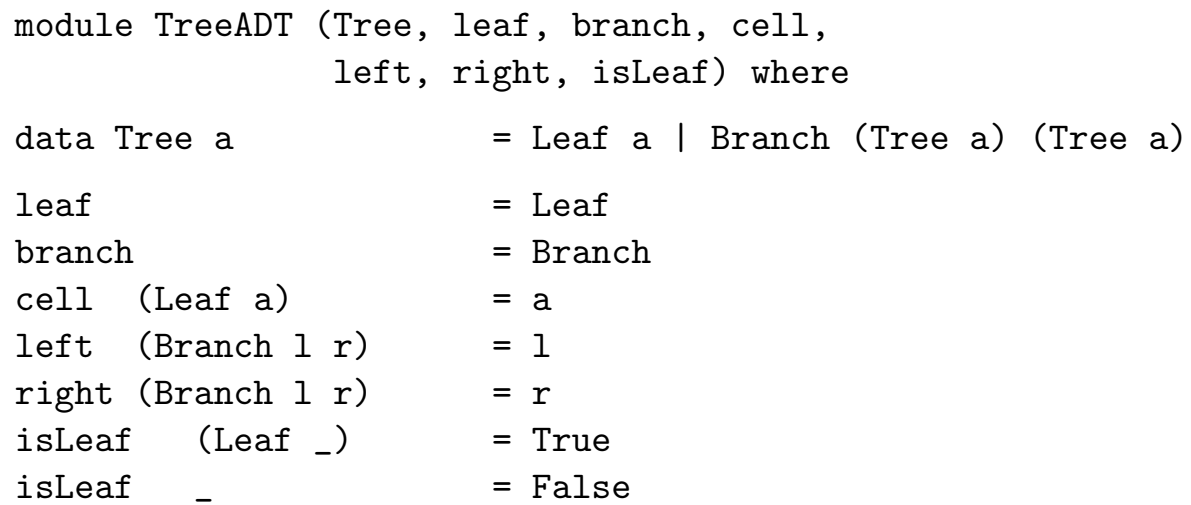

Note in the export list that the type name Tree appears alone (i.e. without its constructors). Thus Leaf and Branch are not exported, and the only way to build or take apart trees outside of the module is by using the various (abstract) operations. Of course, the advantage of this information hiding is that at a later time we could change the representation type without affecting users of the type.

\subsection{More Features}

Here is a brief overview of some other aspects of the module system. See the report for more details.

- An import declaration may selectively hide entities using a hiding clause in the import declaration. This is useful for explicitly excluding names that are used for other purposes without having to use qualifiers for other imported names from the module.

- An import may contain an as clause to specify a different qualifier than the name of the importing module. This can be used to shorten qualifiers from modules with long names or to easily adapt to a change in module name without changing all qualifiers. 
- Programs implicitly import the Prelude module. An explicit import of the Prelude overrides the implicit import of all Prelude names. Thus,

import Prelude hiding length

will not import length from the Standard Prelude, allowing the name length to be defined differently.

- Instance declarations are not explicitly named in import or export lists. Every module exports all of its instance declarations and every import brings all instance declarations into scope.

- Class methods may be named either in the manner of data constructors, in parentheses following the class name, or as ordinary variables.

Although Haskell's module system is relatively conservative, there are many rules concerning the import and export of values. Most of these are obvious - for instance, it is illegal to import two different entities having the same name into the same scope. Other rules are not so obvious - for example, for a given type and class, there cannot be more than one instance declaration for that combination of type and class anywhere in the program. The reader should read the Report for details $(\S 5)$.

\section{Typing Pitfalls}

This short section give an intuitive description of a few common problems that novices run into using Haskell's type system.

\subsection{Let-Bound Polymorphism}

Any language using the Hindley-Milner type system has what is called let-bound polymorphism, because identifiers not bound using a let or where clause (or at the top level of a module) are limited with respect to their polymorphism. In particular, a lambda-bound function (i.e., one passed as argument to another function) cannot be instantiated in two different ways. For example, this program is illegal:

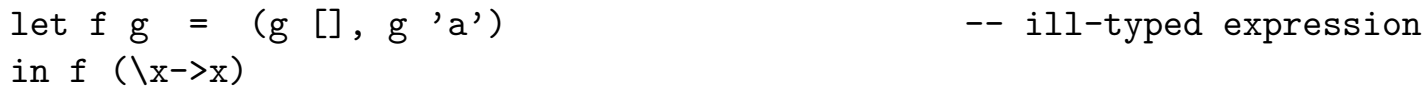

because $g$, bound to a lambda abstraction whose principal type is a->a, is used within $f$ in two different ways: once with type [a]->[a], and once with type Char->Char.

\section{$12.2 \quad$ Numeric Overloading}

It is easy to forget at times that numerals are overloaded, and not implicitly coerced to the various numeric types, as in many other languages. More general numeric expressions sometimes cannot be quite so generic. A common numeric typing error is something like the following:
average xs
$=$ sum $\mathrm{xs} /$ length $\mathrm{xs}$
-- Wrong!

(/) requires fractional arguments, but length's result is an Int. The type mismatch must be corrected with an explicit coercion:

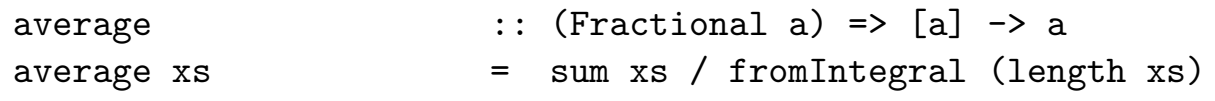




\subsection{The Monomorphism Restriction}

The Haskell type system contains a restriction related to type classes that is not found in ordinary Hindley-Milner type systems: the monomorphism restriction. The reason for this restriction is related to a subtle type ambiguity and is explained in full detail in the Report (§4.5.5). A simpler explanation follows:

The monomorphism restriction says that any identifier bound by a pattern binding (which includes bindings to a single identifier), and having no explicit type signature, must be monomorphic. An identifier is monomorphic if is either not overloaded, or is overloaded but is used in at most one specific overloading and is not exported.

Violations of this restriction result in a static type error. The simplest way to avoid the problem is to provide an explicit type signature. Note that any type signature will do (as long it is type correct).

A common violation of the restriction happens with functions defined in a higher-order manner, as in this definition of sum from the Standard Prelude:

sum

$$
=\text { foldl }(+) 0
$$

As is, this would cause a static type error. We can fix the problem by adding the type signature:

sum $\quad::($ Num a) $\Rightarrow[a] \rightarrow$ a

Also note that this problem would not have arisen if we had written:

sum $\mathrm{xs} \quad=$ foldl $(+) 0 \mathrm{xs}$

because the restriction only applies to pattern bindings.

\section{Arrays}

Ideally, arrays in a functional language would be regarded simply as functions from indices to values, but pragmatically, in order to assure efficient access to array elements, we need to be sure we can take advantage of the special properties of the domains of these functions, which are isomorphic to finite contiguous subsets of the integers. Haskell, therefore, does not treat arrays as general functions with an application operation, but as abstract data types with a subscript operation.

Two main approaches to functional arrays may be discerned: incremental and monolithic definition. In the incremental case, we have a function that produces an empty array of a given size and another that takes an array, an index, and a value, producing a new array that differs from the old one only at the given index. Obviously, a naive implementation of such an array semantics would be intolerably inefficient, either requiring a new copy of an array for each incremental redefinition, or taking linear time for array lookup; thus, serious attempts at using this approach employ sophisticated static analysis and clever run-time devices to avoid excessive copying. The monolithic approach, on the other hand, constructs an array all at once, without reference to intermediate array values. Although Haskell has an incremental array update operator, the main thrust of the array facility is monolithic.

Arrays are not part of the Standard Prelude - the standard library contains the array operators. Any module using arrays must import the Array module.

\subsection{Index types}

The Ix library defines a type class of array indices: 


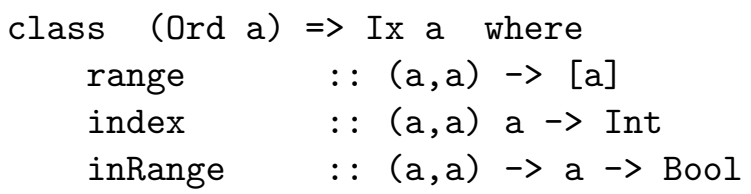

Instance declarations are provided for Int, Integer, Char, Bool, and tuples of Ix types up to length 5; in addition, instances may be automatically derived for enumerated and tuple types. We regard the primitive types as vector indices, and tuples as indices of multidimensional rectangular arrays. Note that the first argument of each of the operations of class Ix is a pair of indices; these are typically the bounds (first and last indices) of an array. For example, the bounds of a 10-element, zero-origin vector with Int indices would be $(0,9)$, while a 100 by 100 1-origin matrix might have the bounds $((1,1),(100,100))$. (In many other languages, such bounds would be written in a form like $1: 100,1: 100$, but the present form fits the type system better, since each bound is of the same type as a general index.)

The range operation takes a bounds pair and produces the list of indices lying between those bounds, in index order. For example,

$$
\begin{gathered}
\text { range }(0,4) \Rightarrow[0,1,2,3,4] \\
\text { range }((0,0),(1,2)) \Rightarrow[(0,0),(0,1),(0,2),(1,0),(1,1),(1,2)]
\end{gathered}
$$

The inRange predicate determines whether an index lies between a given pair of bounds. (For a tuple type, this test is performed component-wise.) Finally, the index operation allows a particular element of an array to be addressed: given a bounds pair and an in-range index, the operation yields the zero-origin ordinal of the index within the range; for example:

$$
\begin{aligned}
\operatorname{index}(1,9) 2 & \Rightarrow \\
\text { index }((0,0),(1,2))(1,1) & \Rightarrow 4
\end{aligned}
$$

\subsection{Array Creation}

Haskell's monolithic array creation function forms an array from a pair of bounds and a list of index-value pairs (an association list):

array $\quad::(\operatorname{Ix} a) \Rightarrow(a, a) \rightarrow[(a, b)] \rightarrow$ Array $a b$

Here, for example, is a definition of an array of the squares of numbers from 1 to 100:

squares $\quad=\operatorname{array}(1,100)[(i, i * i) \mid i<-[1 \ldots 100]]$

This array expression is typical in using a list comprehension for the association list; in fact, this usage results in array expressions much like the array comprehensions of the language Id [6].

Array subscripting is performed with the infix operator !, and the bounds of an array can be extracted with the function bounds:

$$
\begin{aligned}
\text { squares!7 } & \Rightarrow 49 \\
\text { bounds squares } & \Rightarrow \quad(1,100)
\end{aligned}
$$

We might generalize this example by parameterizing the bounds and the function to be applied to each index:

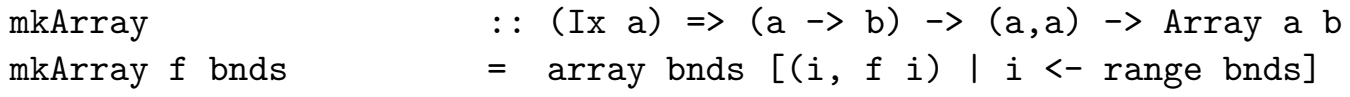

Thus, we could define squares as mkArray ( $i i->i * i)(1,100)$. 
Many arrays are defined recursively; that is, with the values of some elements depending on the values of others. Here, for example, we have a function returning an array of Fibonacci numbers:

fibs : : Int $\rightarrow$ Array Int Int

fibs $\mathrm{n}=\mathrm{a}$ where $\mathrm{a}=\operatorname{array}(0, \mathrm{n})([(0,1),(1,1)]++$

$$
[(i, a !(i-2)+a !(i-1)) \mid i<-[2 \ldots n]])
$$

Another example of such a recurrence is the $n$ by $n$ wavefront matrix, in which elements of the first row and first column all have the value 1 and other elements are sums of their neighbors to the west, northwest, and north:

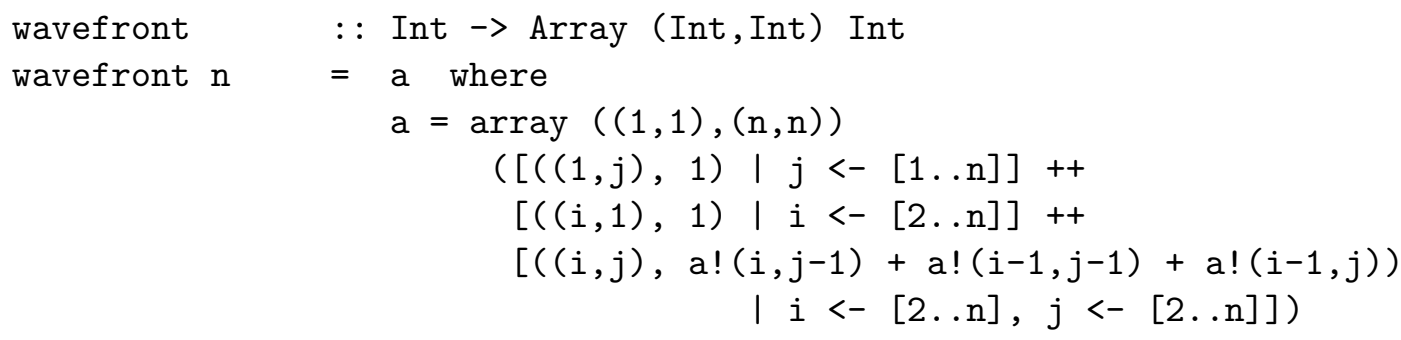

The wavefront matrix is so called because in a parallel implementation, the recurrence dictates that the computation can begin with the first row and column in parallel and proceed as a wedge-shaped wave, traveling from northwest to southeast. It is important to note, however, that no order of computation is specified by the association list.

In each of our examples so far, we have given a unique association for each index of the array and only for the indices within the bounds of the array, and indeed, we must do this in general for an array be fully defined. An association with an out-of-bounds index results in an error; if an index is missing or appears more than once, however, there is no immediate error, but the value of the array at that index is then undefined, so that subscripting the array with such an index yields an error.

\subsection{Accumulation}

We can relax the restriction that an index appear at most once in the association list by specifying how to combine multiple values associated with a single index; the result is called an accumulated array:

accumArray : : (Ix a) $\rightarrow(b \rightarrow c \rightarrow b) \rightarrow b \rightarrow(a, a) \rightarrow[$ Assoc a $c$ ] $\rightarrow$ Array a b

The first argument of accumArray is the accumulating function, the second is an initial value (the same for each element of the array), and the remaining arguments are bounds and an association list, as with the array function. Typically, the accumulating function is $(+)$, and the initial value, zero; for example, this function takes a pair of bounds and a list of values (of an index type) and yields a histogram; that is, a table of the number of occurrences of each value within the bounds:

$$
\begin{array}{ll}
\text { hist } & : \text { (Ix a, Integral } b) \Rightarrow(a, a)->[a]->\text { Array a b } \\
\text { hist bnds is } & =\text { accumArray }(+) 0 \text { bnds }[(i, 1) \mid i<- \text { is, inRange bnds } i]
\end{array}
$$

Suppose we have a collection of measurements on the interval $[a, b)$, and we want to divide the interval into decades and count the number of measurements within each:

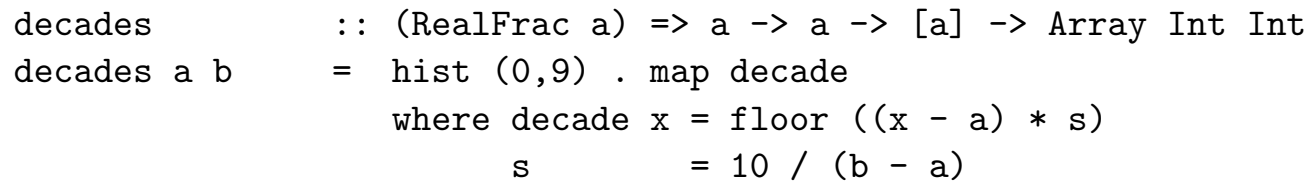




\subsection{Incremental updates}

In addition to the monolithic array creation functions, Haskell also has an incremental array update function, written as the infix operator //; the simplest case, an array a with element $i$ updated to $\mathrm{v}$, is written a // $[(\mathrm{i}, \mathrm{v})]$. The reason for the square brackets is that the left argument of $(/ /)$ is an association list, usually containing a proper subset of the indices of the array:

$$
::(\operatorname{Ix} a) \Rightarrow \text { Array } a b \rightarrow[(a, b)] \rightarrow \text { Array } a b
$$

As with the array function, the indices in the association list must be unique for the values to be defined. For example, here is a function to interchange two rows of a matrix:

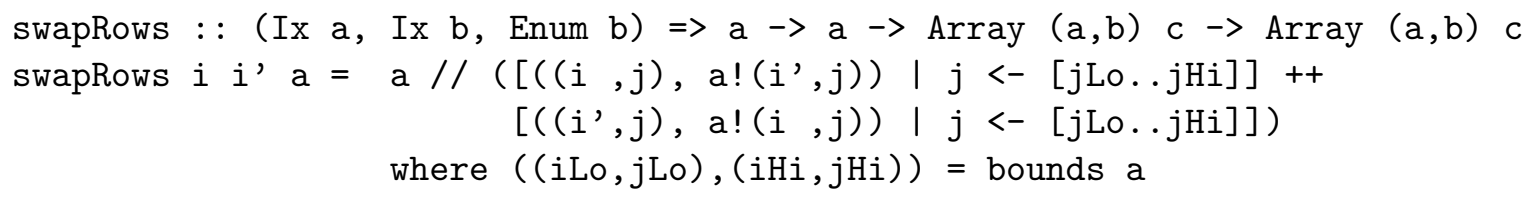

The concatenation here of two separate list comprehensions over the same list of $j$ indices is, however, a slight inefficiency; it's like writing two loops where one will do in an imperative language. Never fear, we can perform the equivalent of a loop fusion optimization in Haskell:

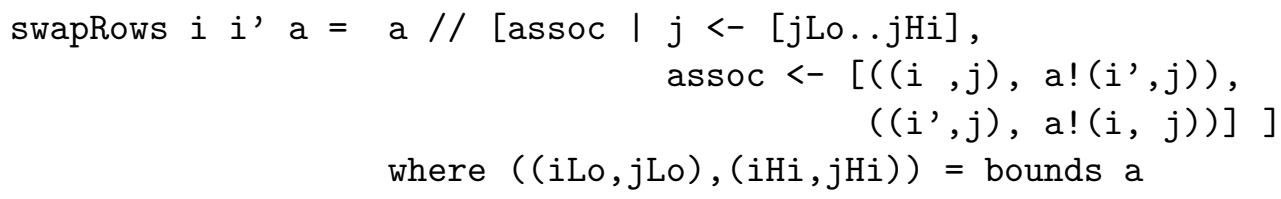

\subsection{An example: Matrix Multiplication}

We complete our introduction to Haskell arrays with the familiar example of matrix multiplication, taking advantage of overloading to define a fairly general function. Since only multiplication and addition on the element type of the matrices is involved, we get a function that multiplies matrices of any numeric type unless we try hard not to. Additionally, if we are careful to apply only (!) and the operations of Ix to indices, we get genericity over index types, and in fact, the four row and column index types need not all be the same. For simplicity, however, we require that the left column indices and right row indices be of the same type, and moreover, that the bounds be equal:

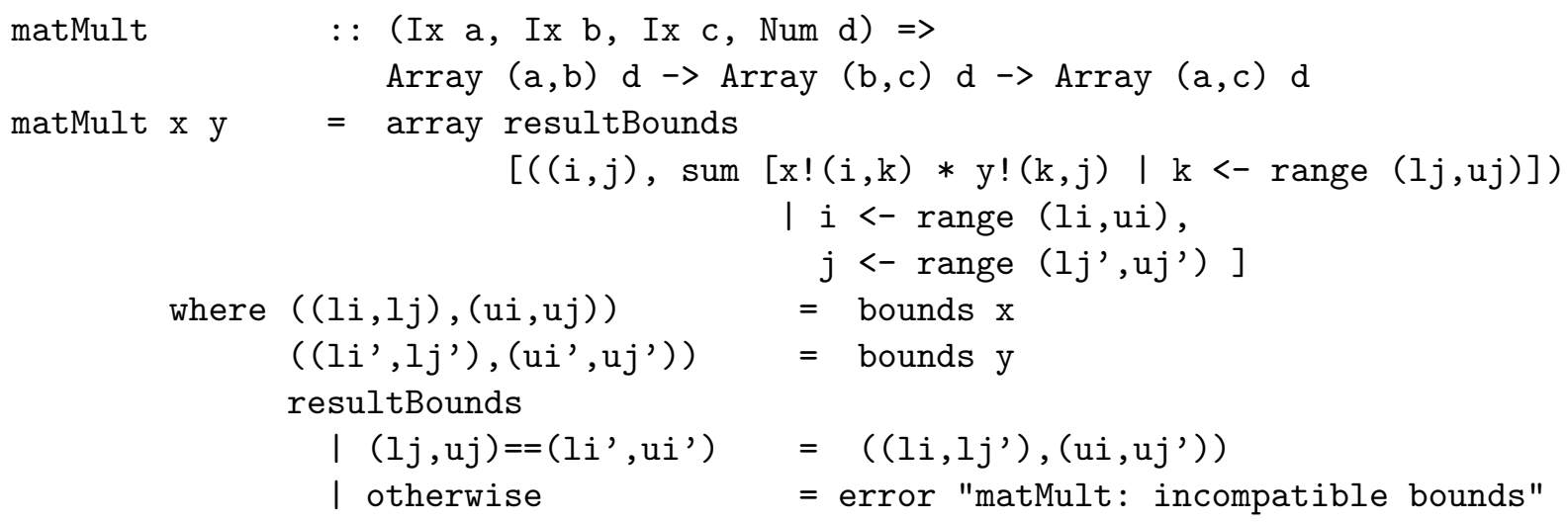

As an aside, we can also define matMult using accumArray, resulting in a presentation that more closely resembles the usual formulation in an imperative language: 


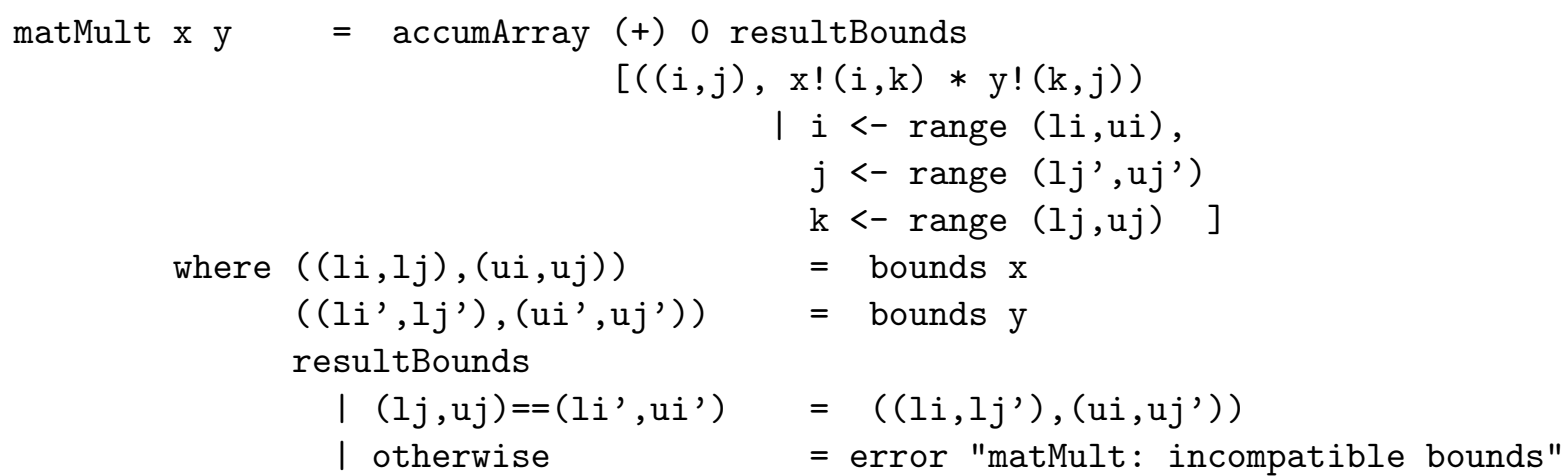

We can generalize further by making the function higher-order, simply replacing sum and (*) by functional parameters:

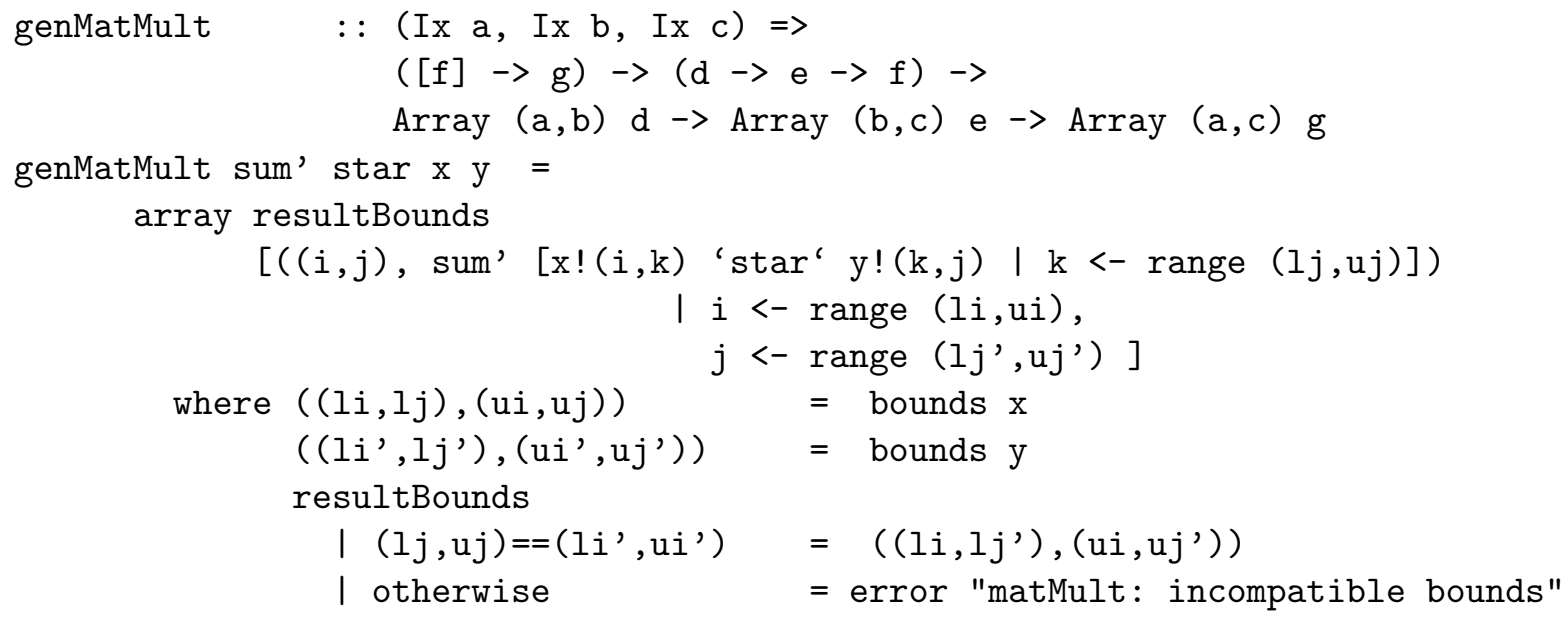

APL fans will recognize the usefulness of functions like the following:

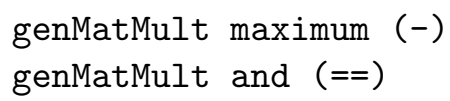

With the first of these, the arguments are numeric matrices, and the $(i, j)$-th element of the result is the maximum difference between corresponding elements of the $i$-th row and $j$-th column of the inputs. In the second case, the arguments are matrices of any equality type, and the result is a Boolean matrix in which element $(i, j)$ is True if and only if the $i$-th row of the first argument and $j$-th column of the second are equal as vectors.

Notice that the element types of genMatMult need not be the same, but merely appropriate for the function parameter star. We could generalize still further by dropping the requirement that the first column index and second row index types be the same; clearly, two matrices could be considered conformable as long as the lengths of the columns of the first and the rows of the second are equal. The reader may wish to derive this still more general version. (Hint: Use the index operation to determine the lengths.)

\section{The Next Stage}

A large collection of Haskell resources is available on the web at haskell.org. Here you will find compilers, demos, papers, and much valuable information about Haskell and functional programming. Haskell compilers or interpreters run on almost all hardware and operating systems. The Hugs system is both small and portable - it is an excellent vehicle for learning Haskell. 


\section{Acknowledgements}

Thanks to Patricia Fasel and Mark Mundt at Los Alamos, and Nick Carriero, Charles Consel, Amir Kishon, Sandra Loosemore, Martin Odersky, and David Rochberg at Yale University for their quick readings of earlier drafts of this manuscript. Special thanks to Erik Meijer for his extensive comments on the new material added for version 1.4 of this tutorial.

\section{References}

[1] R. Bird. Introduction to Functional Programming using Haskell. Prentice Hall, New York, 1998. 1

[2] A.Davie. Introduction to Functional Programming System Using Haskell. Cambridge University Press, 1992. 1

[3] P. Hudak. Conception, evolution, and application of functional programming languages. ACM Computing Surveys, 21(3):359-411, 1989. 1

[4] Simon Peyton Jones (editor). Report on the Programming Language Haskell 98, A Nonstrict Purely Functional Language. Yale University, Department of Computer Science Tech Report YALEU/DCS/RR-1106, Feb 1999. 1

[5] Simon Peyton Jones (editor) The Haskell 98 Library Report. Yale University, Department of Computer Science Tech Report YALEU/DCS/RR-1105, Feb 1999. 1

[6] R.S. Nikhil. Id (version 90.0) reference manual. Technical report, Massachusetts Institute of Technology, Laboratory for Computer Science, September 1990. 13.2

[7] J. Rees and W. Clinger (eds.). The revised ${ }^{3}$ report on the algorithmic language Scheme. SIGPLAN Notices, 21(12):37-79, December 1986. 10

[8] G.L. Steele Jr. Common Lisp: The Language. Digital Press, Burlington, Mass., 1984. 10

[9] P. Wadler. How to replace failure by a list of successes. In Proceedings of Conference on Functional Programming Languages and Computer Architecture, LNCS Vol. 201, pages 113-128. Springer Verlag, 1985. 8.3

[10] P. Wadler. Monads for Functional Programming In Advanced Functional Programming, Springer Verlag, LNCS 925, 1995. 9, 9.3 